\title{
The Influence of the BDNF Val66Met Polymorphism on Mechanisms of Semantic Priming: Analyses with Drift-Diffusion Models of Masked and Unmasked Priming
}

\author{
Alexander Berger', Simon Sanwald', Christian Montag², and Markus Kiefer ${ }^{1}$ \\ 1 Ulm University, Department of Psychiatry, Germany \\ ${ }^{2}$ Ulm University, Department of Molecular Psychology, Germany
}

\section{KEYWORDS}

semantic priming

BDNF Val66Met

drift-diffusion models

masked

unmasked
ABSTRACT

Automatic and strategic processes in semantic priming can be investigated with masked and unmasked priming tasks. Unmasked priming is thought to enable strategic processes due to the conscious processing of primes, while masked priming exclusively depends on automatic processes due to the invisibility of the prime. Besides task properties, interindividual differences may alter priming effects. In a recent study, masked and unmasked priming based on mean response time (RT) and error rate (ER) differed as a function of the BDNF Val66Met polymorphism (Sanwald et al., 2020). The BDNF Val66Met polymorphism is related to the integrity of several cognitive executive functions and might thus influence the magnitude of priming. In the present study, we reanalyzed this data with drift-diffusion models. Drift-diffusion models conjointly analyze single trial RT and ER data and serve as a framework to elucidate cognitive processes underlying priming. Masked and unmasked priming effects were observed for the drift rates $v$, presumably reflecting semantic preactivation. Priming effects on nondecision time to were especially pronounced in unmasked priming, suggesting additional conscious processes to be involved in the to modulation. Priming effects on the decision thresholds a may reflect a speed-accuracy tradeoff. Considering the BDNFVal66Met polymorphism, we found lowered drift rates and decision thresholds for Met allele carriers, possibly reflecting a superficial processing style in Met allele carriers. The present study shows that differences in cognitive tasks between genetic groups can be elucidated using drift-diffusion modeling.

\section{INTRODUCTION}

Access to semantic word meaning can be investigated with the semantic priming paradigm. In semantic priming, the response to a target word is facilitated if it is preceded by a semantically related prime word (Neely, 1991; Neely, 1976). Semantic priming is frequently observed within lexical decision tasks (Meyer \& Schvaneveldt, 1971). This paradigm asks the participants to decide whether a target is an existing word or not. The target words are preceded by either semantically related or unrelated prime words. If the target is preceded by a semantically related word, the response is typically faster and less error prone (Neely, 1991).

Automatic and strategic processes are thought to contribute to semantic priming effects. Possible mechanisms involved in automatic semantic processing are spreading activation (Collins \& Loftus, 1975) and preactivation of semantic features (Masson, 1995; Plaut, 1995).
Spreading activation refers to semantic network models (Collins \& Quillian, 1969). If a concept (e.g., the prime) is processed, the activation of its node in the semantic network spreads to strongly related nodes (e.g., the target), which facilitates processing of the related concepts (Collins \& Loftus, 1975). According to the preactivation of semantic features, it is assumed that the semantic features of the prime are still activated while the target is processed. If there is an overlap of the features associated with the target with those associated with the prime, the meaning of the target can be processed faster (Masson, 1995; Plaut, 1995). Given the assumption of a simultaneous activation of the prime and target properties, these processes are thought to predominate at

Corresponding author: Markus Kiefer, Ulm University, Department of Psychiatry Section for Cognitive Electrophysiology, Leimgrubenweg 12, 89075 Ulm, Germany. E-mail: markus.kiefer@uni-ulm.de 
short stimulus onset asynchronies (SOAs) between the prime and target (Neely, 1991). On the other hand, examples for strategic processes are expectancy generation and semantic matching. Expectancy generation describes a strategy where according to the prime, a set of possible candidate targets is generated. If the following target is part of this set of candidates, the response is facilitated (Neely et al., 1989; Posner \& Snyder, 1975). Semantic matching is thought to occur after the target word has been lexically accessed (Neely et al., 1989). If target and prime word are semantically related, it is likely that the target is a real word facilitating the lexical decision (e.g., den Heyer et al., 1985; Neely, 1977; Seidenberg et al., 1984). To distinguish between different processes involved in semantic priming, the visibility of the prime can be manipulated.

\section{Drift-Diffusion Models}

The drift-diffusion model (Ratcliff, 1978) can be used as a framework to investigate different processes involved in two-choice decision tasks (for an overview, see Voss, Nagler et al., 2013). It offers the advantage of a joint analysis of single trial RT and accuracy data. Drift-diffusion models have already been successfully applied to elucidate the processes underlying priming (e.g., Gomez et al., 2013; Ratcliff \& McKoon, 1988; Voss, Rothermund et al., 2013).

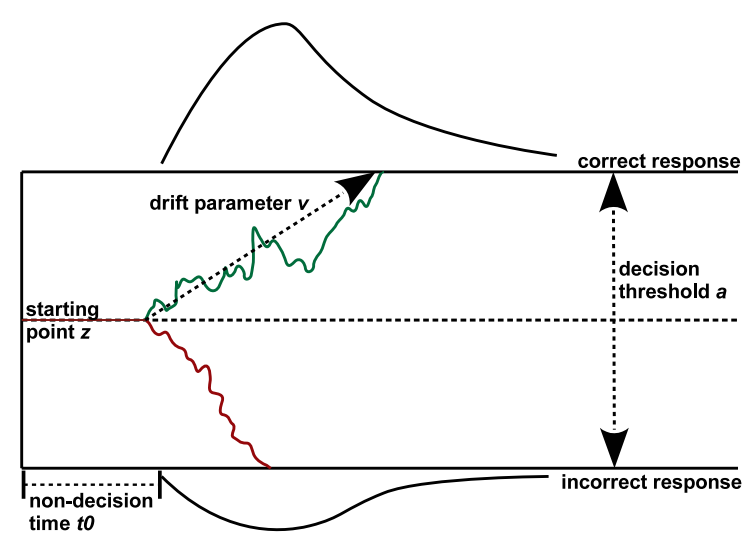

\section{FIGURE 1.}

Visualization of the drift-diffusion model. The drift-diffusion model assumes that information accumulates in a (noisy) drift process until one of two thresholds is reached and the response is initiated. The respective response time (RT) distributions for the correct and incorrect responses are depicted above the upper and below the lower threshold. The drift parameter $v$ represents the average drift rate across trials, that is, the speed of information accumulation. The green and red lines show examples for the drift process of a single trial. The decision threshold a represents the amount of information needed to separate both thresholds. The nondecision time to includes processes not involved in the decision process. These processes can occur before the decision process (e.g., encoding processes) and afterwards (e.g., response execution). Only the nondecision processes before the decision process are shown. The starting point $\mathrm{z}$ is fixed to $a / 2$ in this example; no a priori bias towards one response is expected.
Drift-diffusion models include four main parameters, the drift rate $v$, the decision threshold $a$, the nondecision time $t 0$ and the starting point $z$ (see Figure 1).

The basic assumption of the model is that information accumulates until a decision threshold is reached and the response is initiated (Ratcliff \& McKoon, 2008; Voss et al., 2004; Voss, Nagler, et al., 2013). This information accumulation is called drift and is represented by the drift rate $v$. According to its application to describe two-choice decision tasks, there are two decision thresholds representing the two possible responses. Typical examples for dichotomous decisions require participants to decide between a word and nonword stimuli, for example. Alternatively, dichotomous response categories can be achieved by coding the responses as correct and incorrect. The parameter $a$ represents the distance between both thresholds, that is, the amount of information needed to separate the two decisions. The nondecision time $t 0$ reflects processes not involved in the information accumulation process, for example, perceiving a stimulus or executing the response. It is also possible to vary the starting point $z$, that is, the position between both thresholds where the drift starts, modeling a possible bias towards one response category. Response categories can be set to correct and incorrect responses in tasks where an a priori bias for response alternatives is unlikely. As there cannot be a bias towards a correct response (as correctness is evaluated after the response), $z$ can be fixed to $a / 2$ in such tasks (Voss, Nagler, et al., 2013). In the current study,we only modeled responses to the word targets consistent to the previous analyses of RTs and ERs in Sanwald et al. (2020). Therefore, a different a priori bias towards a response should be ruled out, as the correct response is the same across relatedness conditions (word response). Word targets in the different relatedness conditions were matched for word length and word frequency (Kiefer, 2002; Kiefer et al., 2005), rendering differences in the tendency to respond to these targets between the relatedness conditions unlikely. The drift-diffusion model response categories were therefore set to correct and incorrect responses to word targets (correct response: word; incorrect response: nonword) and $z$ was fixed to $a / 2$.

To gain further insight into the processes which the drift-diffusion model parameters index, one can consider the factors influencing these parameters. The magnitude of drift rate $v$ changed with stimulus difficulty (Ratcliff \& McKoon, 2008; Voss et al., 2004; Voss, Nagler, et al., 2013) and individual differences in working memory and intelligence (Schmiedek et al., 2007). Manipulating the response criterion by different instructions (speed vs. accuracy) influenced the decision threshold $a$ (Ratcliff \& McKoon, 2008; Voss et al., 2004). Another example are slower RTs in elderly people, which were shown to be related to a more conservative response criterion and, therefore, a larger parameter $a$ (Ratcliff et al., 2000, 2006). An elevated difficulty of the motor response (Voss et al., 2004) as well as task switching (Schmitz \& Voss, 2012) increased the nondecision time $t 0$. Encoding processes are also thought to contribute to the processes involved in $t 0$ (Voss, Nagler, et al., 2013). Only a few studies considered the influence of semantic priming on the drift-diffusion model parameters. Furthermore, findings and corresponding interpretations are diverging. One earlier study (Voss, 
Rothermund, et al., 2013) investigated unmasked semantic priming with a lexical decision task. It found an overall effect of semantic relatedness on $v$, indicating an "improved [...] accessibility of targets and their semantic features" (Voss, Rothermund, et al., 2013, p. 553). Additionally, unexpected by the authors, in one of the two experiments using a lexical decision task, to was also shorter for related primes, rendering further processes possible to be included in unmasked priming (Voss, Rothermund, et al., 2013). The $t 0$ modulation in unmasked priming is thought to index conscious processing of primes. Different studies suggested different processes for this effect on $t 0$, that is, the confirmation of the affirmative response in related prime-target pairs (Klauer \& Musch, 2002; Voss, Rothermund, et al., 2013; Wentura, 2000) and a postlexical backward matching strategy (Kiefer et al., 2020; Neely et al., 1989).

In contrast, another study (Gomez et al., 2013) reported that masked priming solely influenced the nondecision time $t 0$, while unmasked priming influenced both $t 0$ and $v$. This pattern indicates $t 0$ is the crucial parameter for priming effects, as $t 0$ was influenced in both masked and unmasked conditions. Following the modulation of $t 0$ in masked priming, which should rule out conscious processes, Gomez et al. hypothesized to to represent an encoding facilitation process. Nevertheless, it remains unclear if masked priming mainly bears on encoding facilitation. Since the priming effect on response times (related-nonrelated primes) for the masked paradigm did not reach significance, Gomez et al. in fact did not observe significant masked priming at all. Therefore, it is unclear how reliable the effects in the masked priming paradigm were. The lack of an effect on $v$ in the masked priming paradigm could be a consequence of the quite high prime duration (56 ms) and the lack of a backward mask. According to this design, participants could have been (partly) aware of the primes, which could diminish priming effects for partly visible masked primes due to an inhibition mechanism (Bodner \& Masson, 2003; Carr \& Dagenbach, 1990; Durante \& Hirshman, 1994; Fischler \& Goodman, 1978).

A more recent study tested the retest reliability of the drift-diffusion model parameters (Lerche \& Voss, 2017), including an assessment of unmasked semantic priming at two time points. Lerche and Voss reported a statistically significant effect on drift rates at both time points. Additionally, there was a small, but not statistically significant effect on the nondecision time, indicating a shortened $t 0$ in related prime-target pairs.

To sum up, semantic relatedness consistently influenced the drift rates in unmasked priming. There are also hints for shortened nondecision times in the related condition in unmasked priming, but there is little agreement on the size of this effect. As, to our knowledge, only one study examined drift-diffusion model parameters in masked semantic priming (Gomez et al., 2013), it is even harder to draw conclusions for the masked priming condition. Table 1 provides an overview of the effect sizes (Lakens, 2013) for all three studies. The priming effects on drift rate (in unmasked priming) show the largest effect sizes, while the effect sizes for the nondecision times vary from small to medium to large. The effect on $t 0$ in unmasked priming and the effect on all parameters in masked priming therefore need further investigation.

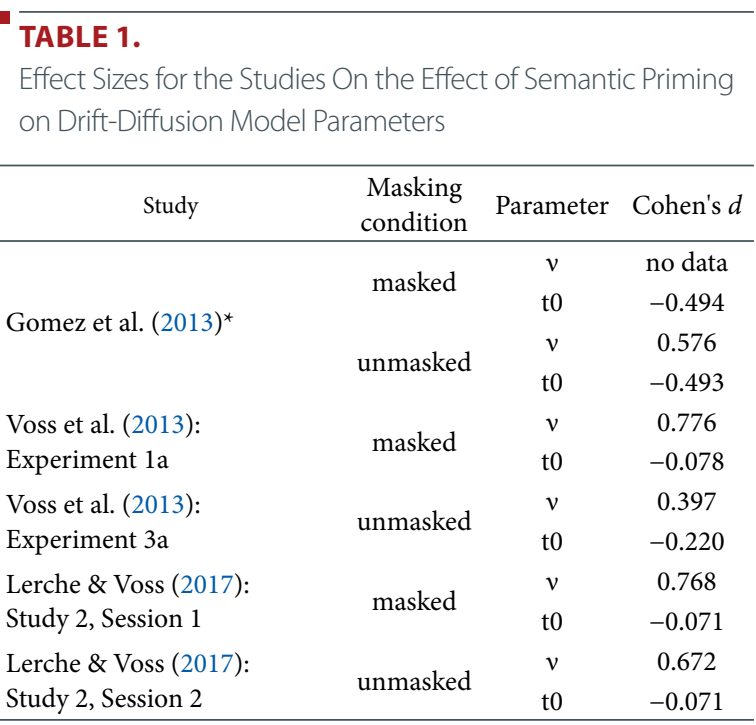

Note. Only results for word targets are shown. Cohen's $d$ effect sizes were calculated by subtracting the related minus the nonrelated conditions and dividing through the mean $S D$ of both conditions.

* Gomez et al. (2013) did not report parameter values and their according SDs. The effect sizes for their paper are therefore calculated using the $t$-values of the reported significance tests with the formula $d=t / \operatorname{sqr} t(N)$ (Lakens, 2013). Also note that the $t$ values for $t 0$ were multiplied with -1 , as Gomez and colleagues reported the comparison of nonrelated-related.

\section{Interindividual Differences in Semantic Priming and Their Relation to Molecular Genetics}

Although unmasked and masked priming effects have been reliably observed in a variety of studies, there are interindividual differences in the magnitude of priming effects, which depend on various factors. For example, priming was shown to be influenced by the integrity of executive functions, showing increased priming for subjects with low executive functions (Kiefer et al., 2005; Moritz et al., 1999), and type of mood states (Hänze \& Hesse, 1993), with good mood associated with increased priming. Priming effects have also been found to be altered in mental disorders such as schizophrenia (Kiefer et al., 2009; Kwapil et al., 1990; Moritz et al., 2003; Spitzer et al., 1993).

In order to elucidate interindividual differences in semantic priming, genetic influences have been considered in the past (Reuter et al., 2009). Based on the assumed relation between executive functions and the magnitude of priming, we recently investigated whether the BDNF Val66Met polymorphism, which was shown to be related to executive functions (Egan et al., 2003), influences semantic priming (Sanwald et al., 2020).

The brain-derived neurotrophic factor (BDNF) is a secretory neurotrophin (Barde et al., 1982) which is thought to play a role in the growth and survival of neurons and the regulation of synaptic transmission (Lu, 2003; Martinowich et al., 2007). The BDNF Val66Met polymorphism manifests in the substitution of the amino acid valine 
(Val) to methionine (Met) at codon 66 of the BDNF molecule (Hall et al., 2003; for the molecular details see Sanwald et al., 2020). The Met allele is thought to be related to impaired BDNF secretion (Egan et al., 2003) and to cognitive impairment in several domains. For example, Met allele carriers were associated with reduced hippocampus volume (Bueller et al., 2006; Montag et al., 2009; Montag, SchoeneBake, et al., 2010) and reduced prefrontal cortex volume (Montag et al., 2009; Pezawas et al., 2004). Semantic priming as well as executive functions showed overlapping neural correlates within the prefrontal cortex (Norman \& Shallice, 1986; Ulrich et al., 2013, Ulrich et al., 2014; Wagner et al., 2001), rendering BDNF Val66Met a potentially influencing candidate, as the projections between hippocampal and prefrontal regions were shown to influence cognition and memory (Laroche et al., 2000). However, note that in the Caucasian population, the Met allele occurs more seldom and, therefore, in many studies $66 \mathrm{Met}+$ carriers (Val/Met and Met/Met combined) are tested versus 66Met- carriers (Val/Val).

However, considering the phenotypic influence of BDNF Val66Met on cognition, the literature shows heterogeneous results. To mention some example findings; the Met allele has been associated with inferior episodic memory (Egan et al., 2003) as well as impaired visual iconic (Beste et al., 2011) and long-term memory (Montag et al., 2014). In contrast, the Met allele was related to reduced Stroop interference (Gajewski et al., 2012) and enhanced task switching (Gajewski et al., 2011). In both tasks (Gajewski et al., 2011, 2012), Met allele carriers showed overall faster RTs and fewer errors, that is, better task performance. As a consequence of such heterogeneous results, there was no meta-analytically determinable influence of BDNF Val66Met on several cognitive phenotypes, including executive functions (Mandelman \& Grigorenko, 2012). Nevertheless, recent meta-analyses investigating the influence of BDNF Val66Met on cognition in more specific domains reported several influences. Met allele carriers showed increased cognitive impairment in patients with Parkinson's disease (Wang et al., 2019; Yin et al., 2019), and an adverse influence of the Met allele on declarative memory and hippocampal volume (Kambeitz et al., 2012). Note that a more recent meta-analysis (Harrisberger et al., 2014) suggested the influence of the Met allele on hippocampal volume to be smaller than reported by Kambeitz et al.. To sum up, the influence of BDNF Val66Met on cognition is still a highly debated field of research.

Given the possible relation of the BDNF Val66Met polymorphism and executive functions, we investigated in an earlier study the influence of this polymorphism on semantic priming including possible moderators (Sanwald et al., 2020). We used masked (Kiefer, 2002) and unmasked (Kiefer et al., 2005) versions of the semantic priming paradigm to analyze individual differences as a function of the BDNF Val66Met polymorphism in unconscious and conscious processing conditions, respectively (see also Reuter et al., 2009). Therefore, as the primary goal was to investigate interindividual differences in priming, and not to directly compare the magnitude of masked versus unmasked priming, both priming paradigms differed. In the masked priming paradigm, directly semantically related and nonrelated prime target pairs were presented, whereas in the unmasked version, an additional condition with indirectly related primes was included to investigate the depth of semantic activation across different semantic distances (McNamara, 1992). An example for an indirectly related prime-target pair is lemon-sweet, which are semantically connected through the mediating link sour. Unmasked priming in the indirectly related condition has been shown to be particular sensitive to differences in working memory and psychopathological states (Kiefer et al., 2005; Spitzer et al., 1993). Furthermore, in the masked version prime duration was very brief $(33.5 \mathrm{~ms})$ to ensure unconscious processing (Breitmeyer, 2007), which was additionally tested with a prime identification test. In the unmasked version, a longer prime duration (200 ms) was used to facilitate conscious prime recognition in correspondence with earlier work (Kiefer et al., 2005; Spitzer et al., 1993). Capacity of executive functions in the participants was tested with a digit span backward test.

In the masked priming paradigm, an influence of the BDNF Val66Met polymorphism on priming was found. However, the pattern of results was reversed for RT and ER data: Met allele carriers showed reduced RT priming, but increased ER priming compared to Val/Val homozygotes. Furthermore, priming effects were not related to differences in executive functions, because BDNF Val66Met groups did not differ in the digit span backward task performance, and digit span performance did not correlate with the magnitude of priming. In the unmasked priming paradigm, performance did not significantly differ between BDNF Val66Met groups, albeit RT and ER differences pointed in the same direction as in the masked version. Given that associations with executive functions were absent, we hypothesized the elevated ER priming and reduced RT priming in the Met allele carriers during masked priming to indicate a more superficial processing style compared to Val/Val homozygotes (Sanwald et al., 2020). We hypothesized that Met allele carriers process the targets more rapidly, but also more erroneously, without indepth semantic analysis. An overall higher ER in Met allele carriers favored this explanation. An analysis of the data with drift-diffusion models should reveal how the observed pattern in RT and ER is reflected by the parameters of the drift-diffusion model. To be more precise, we wanted to assess whether the post-hoc explanation of a superficial processing style of Met allele carriers based on RT and ER data is in line with the results of a drift-diffusion model analysis.

\section{Aim of the Study}

The aim of this study was to decompose the mechanisms underlying the influence of the BDNF Val66Met polymorphism on masked and unmasked semantic priming. We applied drift-diffusion model analyses to the data of our recent study (Sanwald et al., 2020) to elucidate the cognitive processes involved in the semantic priming tasks. Given the diverging priming pattern in the RT and ER data, drift-diffusion model analyses were used to identify the underlying processes. Driftdiffusion model analyses have the advantage of a conjoint analysis of single trial RT and accuracy data and, therefore, might be more sen- 
sitive to reveal influences of the BDNF Val66Met polymorphism on priming also in the unmasked condition.

In both masked and unmasked priming, we hypothesized the drift rate $v$ would be generally influenced by semantic relatedness indicating semantic preactivation (Lerche \& Voss, 2017; Voss, Rothermund, et al., 2013) with larger drift rates in the related conditions. In unmasked priming, drift rates should increase with increasing semantic relatedness from unrelated, over indirect to direct semantic relations. According to larger priming effects in unmasked priming tasks (Kiefer, 2002; Kiefer \& Spitzer, 2000), we expected a larger $v$ modulation in unmasked than in masked priming. As task instruction (Ratcliff \& McKoon, 2008; Voss et al., 2004) and individual response criterion (Ratcliff et al., 2000, 2006) are not supposed to be influenced by masking and semantic relatedness, the decision threshold $a$ should not change over the different masking and relatedness conditions. As the reported influences of semantic relatedness on the nondecisional parameter $t 0$ are diverging (Gomez et al., 2013; Lerche \& Voss, 2017; Voss, Rothermund, et al., 2013), it is difficult to predict the effect on to. Nevertheless, such an effect should manifest in shorter nondecision times in related prime-target pairs. We expected an influence on $t 0$ in particular following conscious prime processing, in which postlexical priming mechanisms can affect response preparation (Kiefer et al., 2020).

Finally, the BDNF Val66Met polymorphisms was expected to influence drift rate $v$ and decision threshold $a$. In line with the hypothesized superficial processing style of Met allele carriers (without indepth lexical analysis), we assumed Met allele carriers would show a general lower decision threshold $a$ and a reduced drift rate $v$. As driftdiffusion model analyses conjointly consider RT and ER data, they might be more sensitive to reveal such differences in both masked and unmasked versions of the priming paradigm.

\section{METHODS}

\section{Participants}

Data from a recent study (Sanwald et al., 2020) was reanalyzed with drift-diffusion models. In total, 188 German participants were recruited from the Ulm Gene Brain Behavior Project (UGBBP) database. All participants gave written informed consent and participated voluntarily. Thirty-three participants were excluded due to the following reasons: Not fulfilling the inclusion criteria of no psychiatric disorder $(n=15)$, no successful genotyping $(n=4)$, extremely high/ low response time $(n=9)$, near chance performance in the masked priming paradigm $(n=1)$, and above chance performance in the prime identification task $(n=4)$. The data of the remaining 155 participants was analyzed. Seventy eight percent of the participants in the included sample were female $(n=121)$. Mean age was 22.23 years $(S D=3.6)$. Participants were mainly students $(74.2 \%) ; 25.2 \%$ had a college or technical college degrees and one participant $(0.62 \%)$ had no school-leaving qualification. The local ethics committee at Ulm University approved this study.

\section{Genotyping}

Participants were genotyped regarding BDNF Val66Met polymorphism. Details of the genotyping process are reported in Sanwald et al. (2020). Due to a low number of participants homozygous for 66Met $(N=7 ; 4.5 \%)$, homozygous 66Met carriers and heterozygous Val/ Met carriers $(N=50 ; 32.3 \%)$ were combined into one group (Met + ). The remaining 98 participants (63.2\%) were homozygous for Val66. These frequencies are comparable to other studies investigating BDNF Val66Met in Caucasian populations (Montag, Basten, et al., 2010; Shimizu et al., 2004).

\section{Procedure}

Before the experiment started, participants completed a German version of the Edinburgh Handedness Inventory (Oldfield, 1971), identifying their response hand. Participants performed a primed lexical decision task in a masked prime and an unmasked prime condition. During the lexical decision task, they were asked to decide whether a target word was a real word or a pseudoword. Pseudowords were not lexically meaningful but appeared like a German word (e.g., Gobel) and served as distractors. All primes were lexically meaningful German words. Participants were instructed to respond as quickly and as accurately as possible. Responses (word vs. pseudoword decision) were given by pressing one of two buttons on a response box by either the index finger (word response) or the middle finger (pseudoword response). Response buttons were laterally reversed depending on the handedness of the participants. The masked priming paradigm was conducted before the unmasked priming paradigm. Participants performed 24 training trials before each priming paradigm started. Presentation and programming of the experiments was carried out using the ERTS (Experimental Run Time System, Berifsoft, Frankfurt, Germany) software.

The masked priming paradigm used in this study was adapted from our previous work (Kiefer, 2002; Kiefer \& Brendel, 2006; Montag et al., 2009). Half of the targets were meaningful words $(n=80)$, the other half were pseudowords $(n=80)$. Fifty percent of the primes preceding a meaningful target word were semantically related to the target $(n$ $=40$, e.g., hen-egg), the other half was non-related to the target (e.g., leaf-car). Nine random letters were used as mask. The sequence of a trial is shown in Figure 2, Panel A.

In the unmasked priming condition, primes were presented for $200 \mathrm{~ms}$ (for the complete sequence of a trial, see Figure 2, Panel B). Concerning the primes preceding a meaningful target, one third ( $n=$ 18) was directly related to the target, one third was indirectly related ( $n$ $=18$, e.g., lemon-sweet), and the remaining third of the primes were nonrelated $(n=18)$. Meaningful targets made up $50 \%$ of the targets $(n=54)$, the other half of the targets were pseudowords $(n=54)$. This paradigm was also adapted from previous work (Kiefer et al., 2005).

To ensure that participants were not aware of the masked primes, a prime recognition task was conducted at the end of the study, that is, after the masked and unmasked priming paradigms. In the recognition task, 80 trials taken from the masked priming paradigm were presented. Primes were words in half of the trials $(n=40)$ and mean- 


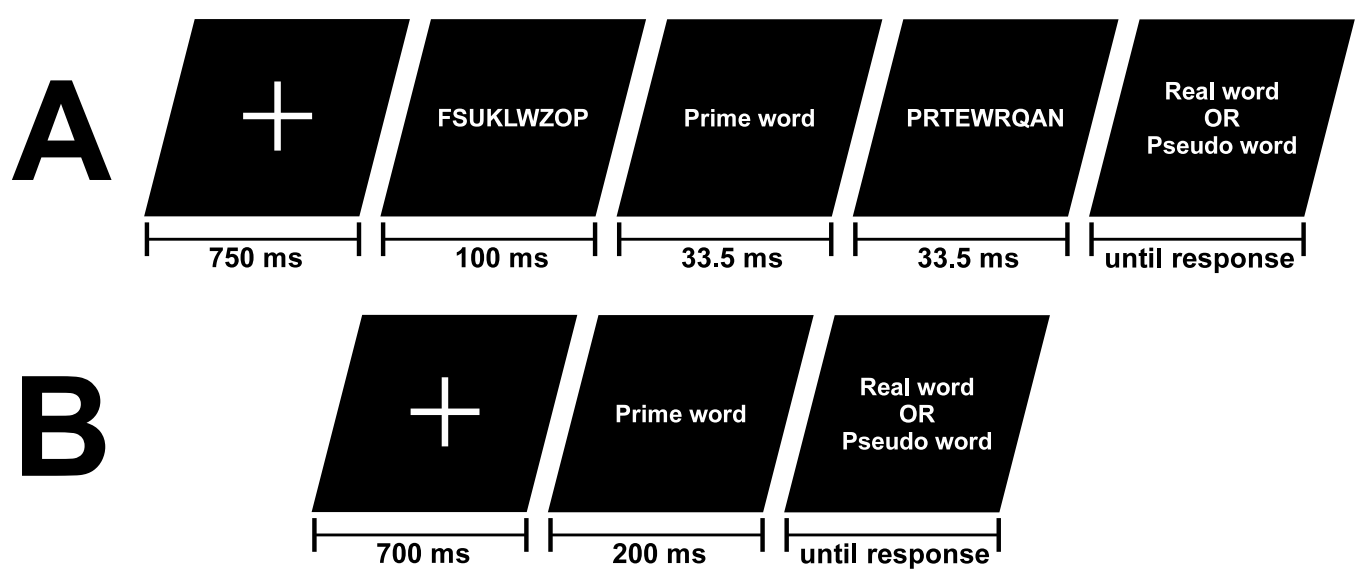

\begin{abstract}
FIGURE 2.
Masked and unmasked priming paradigms. Panel A: Masked priming paradigm. Each trial started with a fixation cross for 750 ms, followed by a forward mask for 100 ms. Afterwards, the prime was presented for 33.5 ms, followed by a backward mask with the same duration. Finally, the target word was presented until the lexical decision was made. Panel B: Unmasked priming paradigm. A fixation cross was presented for $700 \mathrm{~ms}$, followed by the prime for 200 ms. Finally, the target word was presented until the response was given.
\end{abstract}

ingless letter strings ("KKKKKK") in the other half of the trials ( $n=$ 40). The task of the participants was to decide whether the prime was a real word or a meaningless letter string. To evaluate prime awareness, we calculated $d^{\prime}$ measures (Green \& Swets, 1966) for each participant. For this purpose, $z$-transformed relative frequencies of false alarms were subtracted from the z-transformed relative frequencies of hits per participant: $d^{\prime}=z$ (hits) $-z$ (false alarms). Four participants were excluded from the analysis due to above-chance performance (see the Participants section). The other participants performed within the $95 \%$ $\mathrm{CI}$ of chance performance, indicating unawareness of the primes. For further details of the prime recognition task and significance tests considering prime awareness, see Sanwald et al. (2020).

\section{Drift-Diffusion Models}

The RT outliers were excluded if they exceeded +- 2 SDs of the individual mean response time of correct responses per participant separately for the masked and the unmasked condition. If an incorrect response exceeded this threshold, it was excluded as well. Additionally, pseudowords were excluded from the analyses. All remaining responses to the word targets were included in the drift-diffusion model analyses.

Drift-diffusion models were estimated separately for the masked and unmasked condition due to unequal number of trials and the additional indirectly related prime condition in the unmasked priming paradigm. Correct and incorrect responses served as decision thresholds. The models were estimated using hierarchical drift diffusion model (HDDM), version 0.6.0 (Wiecki et al., 2013), a toolbox implemented for Python. The HDDM estimates hierarchical Bayesian parameters of the drift-diffusion model. The starting point $\mathrm{z}$ was fixed to $a / 2$ as an a priori bias towards a correct or incorrect responses is not possible because correctness is determined only after the response (Voss, Nagler, et al., 2013). Intertrial variabilities were fixed to zero. To investigate which parameter combination fits the data best, we estimated each possible parameter combination separately and compared the DIC values (Spiegelhalter et al., 2002; Wiecki et al., 2013) of these models. The DIC (deviance information criterion) is a Bayesian information criterion. Lower values indicate better model fit. The DIC values (see Table 2) indicate a model with $v, a$, and $t 0$ varying for semantic relatedness and BDNF Val66Met group fits the data best, for the masked as well as the unmasked condition. It may seem counterintuitive to vary the decision threshold $a$ depending on semantic relatedness. The driftdiffusion model typically assumes $a$ to be set before the stimulus is shown and relatedness of prime and target should only play a role after the target is shown. Nevertheless, if the threshold was fixed for semantic relatedness, the DIC increased (DIC ${ }_{\text {masked }}=-21926.13$; DIC $_{\text {unmasked }}=$ -13878.10) compared to the model with all parameters freely varying for both conditions. Therefore, we report the results for the model with freely varying drift-diffusion model parameters $v, a$, and $t 0$, and address the interpretation for thresholds varying for relatedness later in the discussion. To achieve stable parameter estimations, 10000 samples were drawn from which 3000 were discarded (parameter estimations usually stabilize after a number of samples large enough). All models converged. Evaluation of model convergence is described in detail in the Supplementary Material A.

\section{Statistical Analysis}

Bayesian repeated-measures ANOVAs were used to analyze the driftdiffusion model parameters. Statistical analyses were performed with JASP, version 0.9.2 (JASP Team, 2019), an open-source statistics program. Bayesian ANOVAs were calculated for each parameter $(v, a, t 0)$ separately for the masked and unmasked conditions by entering the respective subject parameter estimates of the drift-diffusion model into the ANOVA. Genotype was entered as a between-subjects factor (Val/Val and Met+) and semantic relatedness as a within-subject factor (related and unrelated), leading to a $2 \times 2$ design for the masked 
TABLE 2.

DIC scores for the Different Parameter Combinations Separately for the Masked and Unmasked Priming Paradigm

\begin{tabular}{ccc}
\hline Parameter & DIC (masked) & DIC (unmasked) \\
\hline$v$, a, t0 & $\mathbf{- 2 1 9 9 1 . 2 2}$ & $\mathbf{- 1 4 4 4 5 . 3 0}$ \\
v, a & -21803.96 & -14120.82 \\
& & \\
$v$, t0 & -21920.13 & -14389.90 \\
a, t0 & -21950.26 & -14338.35 \\
$v$ & -21852.16 & -14139.78 \\
a & -21761.88 & -14056.17 \\
t0 & -21922.48 & -14353.52 \\
\hline
\end{tabular}

Note. The left column indicates which parameter(s) is / are estimated freely (varying for relatedness and BDNF Val66Met) in the respective model. The DIC score for the best fitting model is depicted in bold.

condition and a $2 \times 3$ design for the unmasked condition (due to the additional relatedness condition of indirectly related). In an additional step, we omitted the indirectly related condition of the unmasked condition and combined the masked and unmasked conditions into one repeated measure Bayesian ANOVA, leading to a $2 \times 2 \times 2$ design (genotype $\times$ semantic relatedness $\times$ masking condition). Due to the different trial numbers between the masked and unmasked condition, this analysis should be treated as an additional exploratory analysis with the purpose of an easier comparison of both masking conditions. To evaluate the strength of the effects, we calculated Cohen's $d$ for the main effects for each drift-diffusion model parameter (for the effect of semantic relatedness averaged across genotype groups and for the effect of genotype averaged across relatedness conditions).

The JASP Bayesian ANOVAs test the experimental factors sequentially by including them step-wise in respective models which are tested against a null model to determine evidence for the inclusion of (a) factor(s) and, therefore, evidence for an effect of the factor(s). This evidence is quantified by a Bayes factor (BF, Rouder et al., 2012), which compares the probability of one model against an alternative model. For example, a BF of 5 indicates that the data is five times more likely given the actual model (e.g., including the investigated factor) compared to an alternative model (e.g., a null model excluding the investigated factor, Rouder et al., 2012). To simplify the comparison of BFs, we report BFs for the effects, that is, for the main effects and the interactions. These BFs compare a model including a specific effect against equivalent models stripped of the effect (Wagenmakers et al., 2018), and can therefore be interpreted as evidence for that effect. To evaluate the evidence for an effect, we used the values recommended by the JASP team (Wagenmakers et al., 2018): a BF from 10-30 indicates strong evidence, a BF from 30-100 indicates very strong evidence and a BF larger than 100 indicates extremely strong evidence for the particular effect. The ANOVA approach was chosen to facilitate the interpretation of the experimental factor effects.

An alternative approach to analyze experimental conditions is to directly compare the posterior group nodes of the relevant parameters.
Direct comparisons were calculated between all conditions per driftdiffusion model parameter and paradigm (masked and unmasked). The probabilities of these differences are directly calculated using the posterior distributions of the respective group parameters (one group parameter per condition). Therefore, the $p$ value indicates the (Bayesian) probability that one parameter is larger (or smaller) than the other (given the data, Wiecki et al., 2013). These comparisons as well as further details of these analyses are provided in the Supplementary Material B. To give a brief overview, these analyses showed a similar result pattern as the Bayesian ANOVA analysis reported below: Despite the related condition in unmasked priming, drift rates and decision thresholds were larger for Val/Val homozygotes than Met+ carriers in all comparisons. Drift rates and decision thresholds were also consistently larger for the related condition(s). Probabilities for a difference between nondecision times in the different relatedness conditions were larger in the unmasked paradigm.

\section{RESULTS}

\section{Descriptive Statistics of Response Times and Error Rates}

Significant effects of semantic relatedness and BDNF Val66Met group (separately for masking conditions) on RTs and ERs were already reported in Sanwald et al. (2020) and are therefore omitted in this article. However, to facilitate the comparison of RTs, ERs, and drift-diffusion model parameters, mean RTs and ERs of the masked and unmasked priming paradigm are shown at a descriptive level (see Table 3 ). There

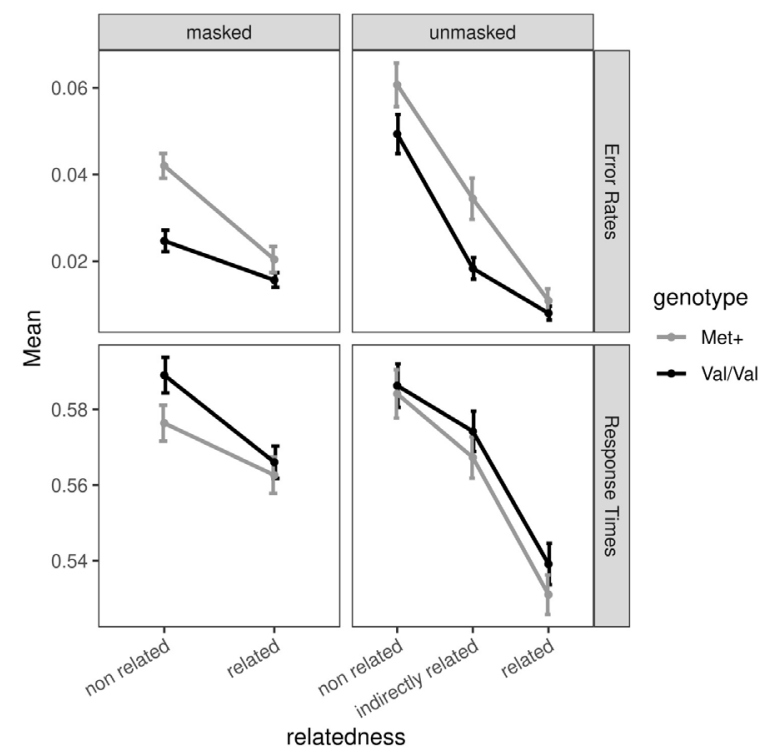

FIGURE 3.

Descriptive statistics. Mean ERs and mean RTs (in seconds) for the masked and unmasked priming paradigms. Note that there was no indirectly related condition in the masked priming paradigm. Val/Nal homozygotes showed consistently slower response times and lower error rates. Error bars show the SEM. 


\begin{tabular}{lccccc}
\hline TABLE 3. & & & \\
Mean Response Times (in Seconds) and Error Rates of the \\
Masked and Unmasked Priming Paradigm
\end{tabular}

were consistent priming effects over all conditions for both RTs and ERs, showing faster RTs and fewer errors in the related compared to the unrelated conditions.

Response times were consistently slower for Val/Val homozygotes compared to Met+ carriers. In contrast, Met+ carriers made more errors across all conditions. The difference between the groups was especially pronounced in the nonrelated condition in the masked priming paradigm (Met+ carriers made considerably more errors and were considerably faster). The descriptive results are visualized in Figure 3.

\section{Masked Priming Paradigm}

Bayesian ANOVAs of the drift-diffusion model parameter values of the masked priming paradigm (see Table 4) revealed several effects. Considering the drift rate $v$, there was extreme evidence for an effect of semantic relatedness, $\mathrm{BF}=4.5^{\star} \mathrm{e}^{41}$, Cohen's $d_{\text {(related - nonrelated) }}=1.51$, and genotype, $\mathrm{BF}=312.3$, Cohen's $d_{(\mathrm{Val} / \mathrm{Val}-\mathrm{Met})}=0.60$. There was no evidence for an interaction of both factors, $\mathrm{BF}=0.2$. These effects manifest in higher drift rates in the related condition as well as higher drift rates for Val/Val homozygotes compared to subjects in the Met+ group. The analysis of the decision thresholds $a$ revealed a main effect of semantic relatedness, $\mathrm{BF}=1.2^{*} \mathrm{e}^{5}$, Cohen's $d_{\text {(related - non-related) }}=0.53$, and genotype, $\mathrm{BF}=1158.6$, Cohen's $d_{\text {(Val/Val }-\mathrm{Met+})}=0.64$, reflecting a larger threshold in the related condition and a larger threshold for Val/Val homozygotes. Additionally, there was strong evidence for an interaction of both factors, $\mathrm{BF}=15.8$, indicating a stronger difference between both genetic groups in the nonrelated condition, (difference ${ }_{(\mathrm{Val} / \mathrm{Val}-\mathrm{Met}+\mid \text { related })}=0.070$; difference $\left._{(\mathrm{Val} / \mathrm{Val} \text { - Met+ } \mid \text { non-related) }}=0.172\right)$. In the nonrelated condition, Met+ carriers showed a substantially lower threshold $a$. In the related condition, the threshold of Met+ carriers was lower as well, but the difference between the genetic groups was smaller. For the nondecision time $t 0$, there was only a main effect of semantic relatedness, $\mathrm{BF}=61.5$, Cohen's $d_{\text {(related - non-related) }}=-0.22$, but no noticeable evidence for an effect of genotype, $\mathrm{BF}=0.3$, Cohen's $d_{(\mathrm{Val} / \mathrm{Val}-\mathrm{Met}+)}=-0.03$, or an interaction of both factors, $\mathrm{BF}=1.1$. The effect of semantic relatedness showed up in a slightly smaller nondecision time $t 0$ in the related condition compared to the unrelated condition (difference (related - nonrelated $\mid \mathrm{Va} / \mathrm{Val})=$ -0.004 ; difference (related - nonrelated $\mid$ Met $)=-0.012$ ).

\section{Unmasked Priming Paradigm}

Parameter values of the drift-diffusion model analysis for the unmasked priming paradigm are shown in Table 5. There was extreme evidence for an effect of semantic relatedness on the drift rate $v$, BF $=9.5^{*} \mathrm{e}^{109}$, Cohen's $d_{\text {(related - nonrelated) }}=3.12$. The drift rates increased as semantic relatedness became stronger (nonrelated to indirectly related to related). Evidence for a main effect of genotype was small, $\mathrm{BF}=4.6$, Cohen's $d_{(\mathrm{Val} / \mathrm{Val}-\mathrm{Met}+)}=0.35$, but there was evidence for an interaction of semantic relatedness and genotype, $\mathrm{BF}=11.7$. The interaction indicates that drift rate was comparable for both genotype groups in the

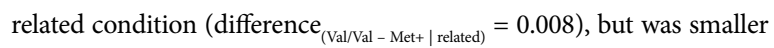
for the Met+ than for the Val/Val group in the indirectly (difference ${ }_{\text {(Val/ }}$ Val - Met+ $\mid$ indirectly related) $=0.287$ ) and the nonrelated condition (difference ${ }_{\text {(Val/ }}$ Val - Met + non-related) $=0.184)$. Regarding the decision threshold $a$, there was extreme evidence for an effect of semantic relatedness, $B F=1.3^{*} \mathrm{e}^{64}$, Cohen's $d_{\text {(related - non-related) }}=1.89$, reflecting higher thresholds for semantically related (and indirectly semantically related) trials. The BF for genotype revealed very strong evidence for an effect, $\mathrm{BF}=135.5$, Cohen's $d_{(\mathrm{Va} / \mathrm{Val} \text { - Met+) }}=0.53$, with elevated thresholds for the $\mathrm{Val} / \mathrm{Val}$ genotype group. Furthermore, there was also extreme evidence for an interaction of semantic relatedness and genotype, $\mathrm{BF}=2.6^{*} \mathrm{e}^{5}$. The interaction arose as consequence of the absence of a difference between the genetic groups in the related condition (difference ${ }_{\text {(Val/Val }}$ Met + related $=-0.001)$, while the decision threshold was smaller for the Met+ group in the indirectly related (difference (Val/Val - Met + indirectly related) $=0.145$ ) and the nonrelated condition (difference (Val/Val - Mett $\mid$ non-related) $=$ 0.097). Finally, there was extreme evidence for a semantic relatedness effect on the nondecision time $t 0, \mathrm{BF}=2.3^{*} \mathrm{e}^{30}$, Cohen's $d_{\text {(related - nonrelated) }}=$

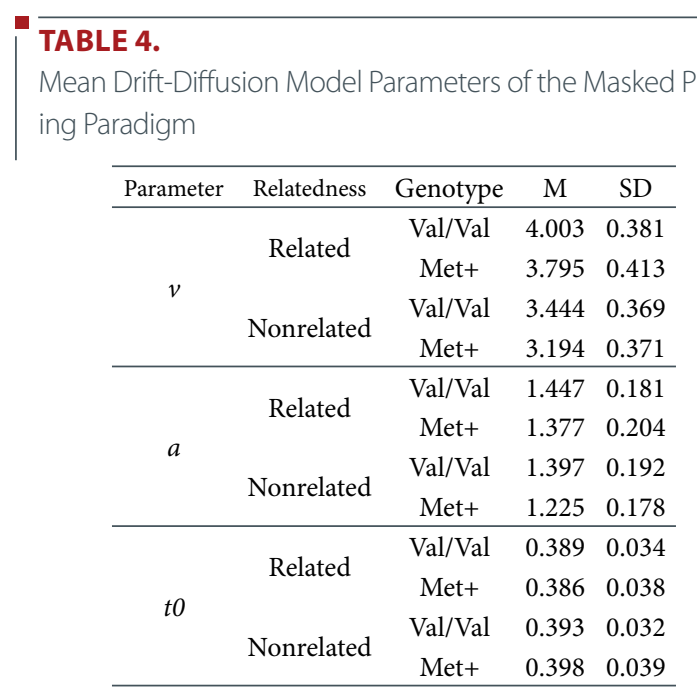




\begin{tabular}{|c|c|c|c|c|}
\hline \multicolumn{5}{|c|}{$\begin{array}{l}\text { TABLE } 5 . \\
\text { Mean Drift-Diffusion Model } \\
\text { Priming Paradigm }\end{array}$} \\
\hline Parameter & Relatedness & Genotype & $M$ & $S D$ \\
\hline \multirow[t]{6}{*}{ - } & \multirow{2}{*}{ Related } & $\mathrm{Val} / \mathrm{Val}$ & 4.427 & 0.480 \\
\hline & & Met+ & 4.419 & 0.493 \\
\hline & \multirow{2}{*}{$\begin{array}{l}\text { Indirectly } \\
\text { related }\end{array}$} & $\mathrm{Val} / \mathrm{Val}$ & 3.808 & 0.416 \\
\hline & & Met+ & 3.521 & 0.536 \\
\hline & \multirow{2}{*}{ Nonrelated } & $\mathrm{Val} / \mathrm{Val}$ & 3.131 & 0.425 \\
\hline & & Met+ & 2.947 & 0.362 \\
\hline \multirow{6}{*}{$a$} & \multirow{2}{*}{ Related } & $\mathrm{Val} / \mathrm{Val}$ & 1.485 & 0.145 \\
\hline & & Met+ & 1.486 & 0.148 \\
\hline & \multirow{2}{*}{$\begin{array}{l}\text { Indirectly } \\
\text { related }\end{array}$} & $\mathrm{Val} / \mathrm{Val}$ & 1.442 & 0.149 \\
\hline & & Met+ & 1.297 & 0.140 \\
\hline & \multirow{2}{*}{ Nonrelated } & $\mathrm{Val} / \mathrm{Val}$ & 1.239 & 0.159 \\
\hline & & Met+ & 1.142 & 0.172 \\
\hline \multirow{6}{*}{ to } & \multirow{2}{*}{ Related } & $\mathrm{Val} / \mathrm{Val}$ & 0.368 & 0.038 \\
\hline & & Met+ & 0.361 & 0.041 \\
\hline & \multirow{2}{*}{$\begin{array}{l}\text { Indirectly } \\
\text { related }\end{array}$} & $\mathrm{Val} / \mathrm{Val}$ & 0.385 & 0.040 \\
\hline & & Met+ & 0.384 & 0.052 \\
\hline & \multirow{2}{*}{ Nonrelated } & $\mathrm{Val} / \mathrm{Val}$ & 0.400 & 0.046 \\
\hline & & Met+ & 0.401 & 0.048 \\
\hline
\end{tabular}

-0.83 , reflecting an increase in $t 0$ with decreasing semantic relatedness $(t 0[$ related $]<t 0$ [indirectly related $]<t 0[$ non related $])$. Regarding the main effect of genotype, $\mathrm{BF}=0.3$, Cohen's $d_{(\mathrm{Val} / \mathrm{Val}-\mathrm{Met})}=0.05$, and the interaction, $\mathrm{BF}=0.1$, there was no evidence for an effect on $t 0$.

\section{Conjoint Analysis of the Masked and Unmasked Priming Paradigms}

To facilitate the comparison of the masked and unmasked priming paradigms, we combined the drift-diffusion model parameters of the masked and unmasked conditions into a conjoint analysis (by excluding the indirectly related condition of the unmasked priming paradigm).

This Bayesian ANOVA revealed extreme evidence for a main effect of semantic relatedness, $\mathrm{BF}=5.6^{*} \mathrm{e}^{114}$ and the interaction of semantic relatedness and masking condition, $\mathrm{BF}=4.1^{*} \mathrm{e}^{40}$, for the drift rate $v$. There was also evidence for an effect of genotype, $\mathrm{BF}=12.6$, and masking condition, $\mathrm{BF}=20.4$, but no convincing evidence for the remaining interactions, all $\mathrm{BFs}<2.6$. Taken together, these results strongly indicate that the drift rate was higher in the related condition and that this difference was particularly pronounced in the unmasked priming paradigm (see Figure 4). That is, the relatedness effect was twice as large in the unmasked than in the masked priming paradigm, $d(v)_{\text {unmasked }} d d(v)$ masked $=2.07$. Considering the genotype effect, Val/Val carriers showed overall larger drift rates compared to Met+ carriers. The results for the decision threshold $a$ indicated extreme evidence for an effect of semantic relatedness, $\mathrm{BF}=3.6^{*} \mathrm{e}^{45}$, for the interaction of semantic relatedness and masking condition, $\mathrm{BF}=3.1^{*} \mathrm{e}^{17}$, and for the interaction of semantic relatedness and genotype, $\mathrm{BF}=6452.0$. Additionally, there was evidence for a main effect of genotype, $\mathrm{BF}=99.6$, and for the interaction of genotype and masking condition, $\mathrm{BF}=45.0$. Considering the main effect of masking condition, $\mathrm{BF}=6.0$, and the three-way interac- tion, $\mathrm{BF}=0.2$, there was comparably little evidence for an effect. To summarize these findings, the decision thresholds were shown to be elevated for related primes and the difference between related and nonrelated condition was larger in the unmasked priming paradigm, $d(a)$ unmasked $/ d(a)_{\text {masked }}=3.53$. In addition, the overall smaller thresholds for the Met+ carriers were less pronounced in the related conditions (see Figure 4). The difference between genotype groups was slightly larger in masked than in unmasked priming, $d(a)_{\text {unmasked }} / d(a)_{\text {masked }}=0.82$. For the remaining drift-diffusion model parameter, the nondecision time $t 0$, the analysis showed extreme evidence for an effect of semantic relatedness, $\mathrm{BF}=7.8^{\star} \mathrm{e}^{20}$, and an interaction of semantic relatedness and masking condition, $\mathrm{BF}=1.1^{*} \mathrm{e}^{10}$. Furthermore, there was also extreme evidence for a main effect of masking condition, $\mathrm{BF}=626.4$. The Bayes Factors of all remaining effects indicated no evidence, all $\mathrm{BFs}<0.8$. As can be seen in Figure 4 , the effects on $t 0$ emerged as a consequence of a strong semantic relatedness effect in the unmasked priming paradigm. Particularly in the unmasked priming paradigm, to was considerably shortened in trials with related primes, that is, the semantic relatedness effect was nearly four times larger in the unmasked than in the masked priming paradigm, $d(t 0)_{\text {unmasked }} / d(t 0)_{\text {masked }}=3.72$.

\section{DISCUSSION}

The present study aimed at elucidating mechanisms underlying the differential RT and ER priming effects as a function of the BDNF Val66Met

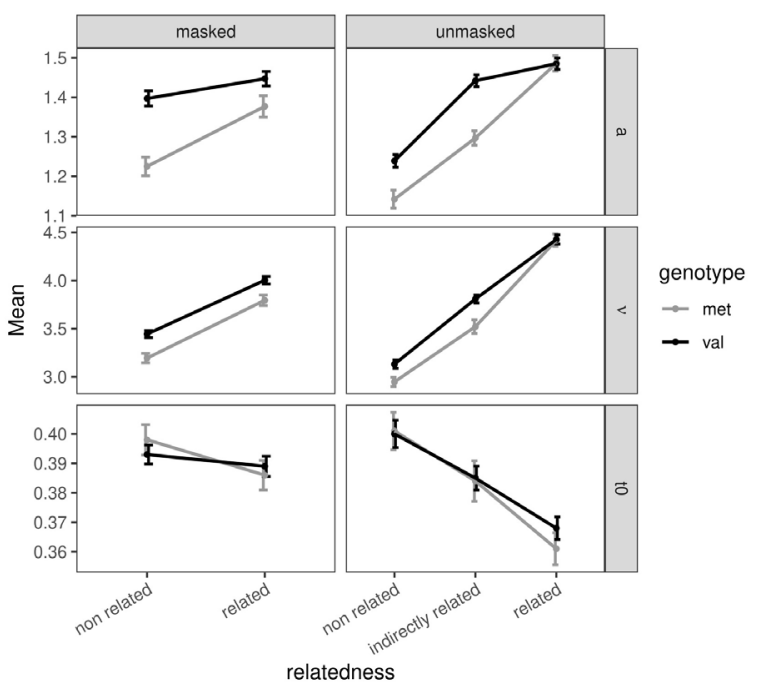

FIGURE 4.

Mean drift-diffusion model parameter for the masked and unmasked priming paradigm. The first row shows the decision thresholds a, the second the drift rates $v$ and the third one the nondecision times $t 0$. The relatedness conditions are depicted on the $x$ axis. Note that there was no indirectly related condition in the masked priming paradigm. Drift rates $v$ and decision thresholds a were elevated in the related conditions. In the unmasked paradigm, nondecision time to was smaller in the related conditions. Met+-carrier showed lowered parameters $v$ and $a$. Error bars show the SEM. 
polymorphism in our recent study (Sanwald et al., 2020): RT and ER masked priming effects were reversed in the different genotype groups, while significant effects were absent in the unmasked priming paradigm. Drift-diffusion models served as framework to analyze (cognitive) mechanisms underlying this complex priming pattern. The present analysis with drift-diffusion models indicated priming effects independently of the genotype on all model parameters in both masked and unmasked priming: Drift rate $v$ and the decision threshold $a$ were higher in the related than in the unrelated condition. The nondecisional parameter to was smaller in the related condition, particularly in unmasked priming, whereas in the masked condition, effect sizes of priming on $t 0$ were small. The BDNF Val66Met polymorphism influenced priming effects with regard to the decision threshold $a$ and the drift rate $v$ in the masked priming paradigm. Most notably, although significant genotype effects were absent in the conventional RT and ER analyses, analyses with drift-diffusion models also revealed influences of the BDNF Val66Met polymorphism on $a$ and $v$ in the unmasked priming paradigm, albeit with smaller effect sizes than in masked priming. Before we return to individual differences in priming as a function of genotype, we discuss general priming effects on model parameters in more detail.

\section{Influence of Semantic Relatedness in Masked and Unmasked Priming Conditions}

The presently observed priming effect on drift rate $v$ (elevated $v$ in the related condition) is in line with studies by Lerche and Voss (2017) and Voss, Rothermund et al. (2013), who identified the drift rate $v$ as a critical parameter in semantic priming. Modulation of $v$ by priming is assumed to reflect semantic preactivation of the semantic target representation by the prime (Voss, Rothermund, et al., 2013; Wentura et al., 2020). In contrast to present effects of priming on drift rate $v$ in both masked and unmasked priming, in the earlier study by Gomez et al. (2013), priming effects on drift rate were absent for masked primes. They reported a consistent influence of semantic relatedness across masked and unmasked priming only on $t 0$. To be more precise, Gomez et al. reported a priming effect on $v$ solely in the unmasked priming paradigm, while t 0 was influenced in both masked and unmasked paradigms. However, there are several differences between the present study and Gomez et al. In Gomez et al, masked RT priming effects were not statistically reliable. Furthermore, due to the longer prime duration, the presence of only a forward mask, and a missing prime awareness assessment, it remains open whether participants were aware of the masked primes in Gomez et al. Awareness of primes at short SOAs may be distracting for the target processing, conceivably interfering with priming effects (Bodner \& Masson, 2003; Durante \& Hirshman, 1994; Fischler \& Goodman, 1978). In the present study, where participants with above-chance performance in the prime identification task were excluded, performance in the prime awareness test did not correlate with any priming effects (related-nonrelated condition) on the drift-diffusion model parameters in the masked priming paradigm (all $|r|<0.08$, all $p s>0.33$ ). These results support the assumption that potential residual prime awareness did not affect masked priming effects. Otherwise, increasing prime awareness should be associated with increased masked priming effects. Nevertheless, similar to Gomez et al. (2013), we observed a small priming effect on to in masked priming, presumably representing a shortened encoding process in related primes (Gomez et al., 2013; Voss, Nagler et al., 2013). However, the magnitude of this effect is considerably smaller than the effect on the drift rate (masked priming paradigm: $d(t 0)=-0.22, d(v)$ $=1.51$ ), therefore indicating the drift rate to be the critical parameter in masked semantic priming.

Both drift rate and $t 0$ priming effects were more pronounced in unmasked priming, in line with larger priming effects in unmasked priming compared to masked priming in the literature (Kiefer, 2002; Kiefer \& Spitzer, 2000). The difference between masked and unmasked priming effects was especially strong for $t 0$. The priming effect on $t 0$ showed the largest increase in unmasked priming compared to masked priming, showing a 3.7 times larger effect. In comparison, the priming effect on the drift rate was about twice as large in unmasked compared to masked priming. This is clearly a consequence of the small priming effect on $t 0$ in masked priming, while the drift rate showed a strong modulation in the masked condition. The transition of a small effect size in masked to priming to a large effect size in unmasked to priming, although all effect sizes were generally smaller compared to the drift rate, is of particular interest when considered in view of the literature. Priming effects on $t 0$ were found in unmasked priming (Voss, Rothermund, et al., 2013) and in masked priming only at long prime durations (Kiefer et al., 2020), that is, presumably following conscious prime processing. In line with this research, an additional mechanism contributing to the enhanced to priming effects in unmasked priming could be considered. One possible mechanism is a fostered affirmative response in related prime-target pairs, which accelerates responding (Klauer \& Musch, 2002; Voss, Rothermund, et al., 2013; Wentura, 2000). In contrast, if prime and target are nonrelated, subjects might take longer for the target response, as prime and target are incongruent. A second possible mechanism could be a postlexical backward matching strategy (Kiefer et al., 2020; Neely et al., 1989). If prime and target are related, the target has to be a semantically meaningful word, facilitating the target response, which is therefore executed faster. As these mechanisms are thought to depend on conscious processing, this might explain why t0 modulations were mainly observed in unmasked priming. An encoding facilitation process (Gomez et al., 2013) seems to play a role in the $t 0$ modulation as well (as the observed small priming effect on $t 0$ in the masked paradigm suggests), but might be less informative in separating conscious and unconscious processing.

Furthermore, the results of the unmasked priming task showed a gradual change of drift rates and nondecision times according to semantic relatedness. The magnitude of the drift rate $v$ increased from nonrelated over indirectly related to directly related prime-target pairs, while $t 0$ was shortened with increasing semantic relatedness, suggesting a similar influence of associative strength on both processes.

The decision threshold $a$ was elevated in the related condition in masked and unmasked priming compared to the unrelated condition. This effect was unexpected and not hypothesized. How conservatively subjects respond to targets should not be affected by semantic related- 
ness, but rather remain constant within an experiment. However, model comparisons using the DIC criterion indicated that model fit was better if the decision threshold $a$ was allowed to vary with regard to semantic relatedness, compared to a model, in which decision threshold was held constant for relatedness. On the behavioral performance level, participants made more errors if prime and target were nonrelated. On the model level, decision thresholds were lowered in nonrelated conditions, indicating participants to respond less carefully in these conditions. In contrast, in the related condition, subjects responded faster and less error prone, reflected by elevated drift rates and elevated decision thresholds. As subjects showed consistent RT priming, that is, faster responses in related conditions, the drift rate elevation had to be large enough to compensate for the increased decision thresholds and, therefore, the more conservative response criterion. Such a dissociation between drift rate and decision threshold could be indicative for occasionally observed dissociations between RT and ER priming in the priming literature. Lack of RT or ER priming could be a consequence of different response strategies. According to the framework of speed-accuracy tradeoffs (Heitz, 2014), a response strategy focused on speed should foster ER priming (by diminishing RT differences), while a strategy focused on accuracy and, therefore, low ERs should boost RT priming. In view of the observed results, that is, lowered decision thresholds in the nonrelated condition, we would assume a premature abortion of evidence accumulation in this condition. The task instruction (Ratcliff \& McKoon, 2008; Voss et al., 2004), that is, to respond as fast and as accurate as possible, may have promoted a response in the nonrelated condition despite an unfinished information accumulation process. As drift rates were lower in the nonrelated condition and, therefore, information accumulated more slowly, subjects may implicitly have recognized their reduced response speed. As a consequence, they possibly initiated their response earlier in the information accumulation process to achieve comparable response speeds across the whole task (leading to lowered decision thresholds in the nonrelated condition), in order to be compliant with the instruction stressing both speed and accuracy. Possibly, participants adopt decision thresholds dynamically during the course of one trial, in order to meet an internal response speed criterion. At least trialwise, changes with regard to caution in responding have been already observed in other domains such as conflict (Kerns et al., 2004) or error processing (Dutilh et al., 2012). A dynamic adaptation of the decision threshold thus might extend semantic priming. Of course, as an influence of semantic relatedness on the decision threshold was unexpected, this interpretation is clearly post-hoc and deserves further investigation. Nevertheless, the assumed influence through a speed-accuracy tradeoff additionally provides an explanation why the priming effect on $a$ is more pronounced in the unmasked priming paradigm. Given the larger priming effect on $v$ in unmasked priming and, therefore, a larger difference in the information accumulation speed between related and nonrelated conditions, subjects had to abort the information accumulation process in the nonrelated condition of unmasked priming even earlier to achieve comparable response speeds across relatedness conditions. This influence was observable across all semantic distances, comparable to the results for the drift rates in unmasked priming.

\section{Interindividual Differences in Priming as a Function of the BDNF Val66Met Polymorphism}

The BDNF Val66Met polymorphism influenced the drift rate $v$ and the decision threshold $a$ in both priming paradigms. The Met+ group showed a lower drift rate as well as lowered thresholds compared to the $\mathrm{Val} / \mathrm{Val}$ group. The difference of decision thresholds between genetic groups was particularly pronounced in the nonrelated condition in the masked paradigm with Met+-carriers showing an especially low decision threshold. Hence, drift-diffusion model analyses revealed separable effects of the BDNF Val66Met polymorphism on the mechanisms underlying priming. To recapitulate, Met+ carriers showed decreased RT priming, but increased ER priming due to an especially high ER in the nonrelated condition. In line with the descriptive result pattern of faster RTs and elevated ERs in the Met+-group (in masked and unmasked priming), we hypothesized Met+ carriers to use a superficial processing style in the lexical decision task, bypassing indepth lexical processing (Sanwald et al., 2020). The lowered drift rates in Met+ carriers indicate a slower information accumulation in the Met+ group (Ratcliff \& McKoon, 2008; Voss, Nagler, et al., 2013), presumably representing less attention to concept meaning due to their superficial processing style. Nevertheless, BDNF Val66Met did not modulate the priming effect on the drift rate; the observed genotype effect on drift rates was a main effect comparable across relatedness conditions. According to the decreased drift rates in Met+ carriers, the faster RTs must arise as consequence of the lowered decision thresholds in the Met+ group because the lowered drift rates would result in slower responses. This effect was more than compensated for by the decreased thresholds. Following this interpretation, Met+-carriers rapidly responded to the targets without paying particular attention to accuracy. Speed versus accuracy tradeoffs (Heitz, 2014) were already shown to influence the decision thresholds (Ratcliff \& McKoon, 2008; Voss et al., 2004). As a consequence, Met+carriers could achieve even faster RTs than the Val/Val homozygotes in exchange for higher ERs. From a motivational point of view, Met+ carriers might have realized their comparable low evidence accumulation (indicated by lower drift rates), leading to frustration. Frustration was shown to be related to reduced decision thresholds, possibly to finish the frustrating task earlier (Lerche et al., 2018). It is possible that (in accordance to the lower drift rates) the task was generally more demanding for Met+-carriers, therefore increasing frustration throughout the experiment. To sum up, according to their hypothesized superficial processing style, Met+ carriers accumulated lexical information including concept meaning more slowly, indicated by decreased drift rates (Voss, Rothermund, et al., 2013; Wentura et al., 2020; for preactivation effects on $v$ in other domains also see Klauer et al., 2007). Additionally, Met+ carriers formed a decision based on insufficient evidence, possibly due to frustration, indicated by lowered decision thresholds compared with Val/Val homozygotes, resulting in faster RTs and higher ERs.

A comparison of the differences of the BDNF Val66Met groups between the two masking conditions showed smaller differences in the unmasked priming paradigm, which were in fact absent in the related condition of the unmasked paradigm. This could be due to a ceiling 
effect. In the related condition of the unmasked paradigm, the overall fastest RTs and lowest ERs were observed. Therefore, performance could have been at maximum in this condition, so that a possible difference between the groups could not be observed. Nevertheless, unlike in the conventional analysis (Sanwald et al., 2020), we observed a difference in drift rates and decision thresholds between BDNF Val66Met groups in the unmasked paradigm (except of the related condition), suggesting an improved sensitivity of drift-diffusion models to detect differences in (cognitive) processes, presumably due to the conjoint analysis of RTs and ERs.

Our drift diffusion model analyses indicated that Met+ carriers accumulated semantic information more slowly (decreased drift rate) and responded based on less accumulated evidence (lowered decision threshold, particularly in the nonrelated condition). Nevertheless, it is unclear how these findings relate to differences in executive functions, which are known to influence the magnitude of priming (Kiefer et al., 2005; Moritz et al., 1999). Given the fact that we observed no difference in executive functions between the BDNF Val66Met groups (Sanwald et al., 2020) as measured with a digit span backward task, a contribution of this cognitive function to the presently observed priming differences is not very likely. To complement this, we calculated correlations between performance in the digit span backward task and the estimated driftdiffusion model parameters. This analysis revealed no significant correlations (all $r s \leq 0.07$, all $p s>0.38$ ). Furthermore, evidence with regard to the influence of the BDNF Val66Met polymorphism on executive functions is heterogeneous. As indicated by a meta-analysis (Mandelman \& Grigorenko, 2012), some studies revealed elevated executive functions in Met+ carriers, while other studies reported decreased executive functions in this group. As a result, the reported meta-analytically determined effect was not distinguishable from zero. As we did not measure other cognitive abilities besides executive functions, it remains unclear which more general cognitive factors could explain the observed differences between BDNF Val66Met groups in semantic priming in the present study.

\section{Limitations}

There are several limitations which have to be considered. First of all, the masked and unmasked priming paradigms differed. The unmasked priming paradigm included fewer trials, a longer SOA and an additional relatedness condition with indirectly related primes. Furthermore, the presentation order of both paradigms was not balanced; participants always performed the masked paradigm first. The direct comparison of masked and unmasked priming paradigm is therefore exploratory and should be interpreted with caution. Investigating the influence of the Met allele was complicated, as our sample was too small to model the Met/ Met-homozygotes separately. The prevalence of Met/Met-homozygotes is about less than $5 \%$ in the Caucasian population (Montag, Basten, et al., 2010; Shimizu et al., 2004) and might therefore require even larger samples (Montag \& Reuter, 2014). In general, complex psychological phenotypes and cognitive processes involved in priming and related constructs are influenced by hundreds or thousands of genetic variants, all with tiny effect sizes (Montag et al., 2020). Therefore, tremendous challenges still lie ahead to understand the complex molecular underpinning of priming. Finally, we observed overall low ERs in the priming paradigms. Low ERs may not be optimal for estimating drift-diffusion models (Ratcliff \& McKoon, 2008; Voss, Nagler, et al., 2013).

\section{Conclusions}

To summarize, the drift-diffusion model analysis provided further insight into mechanisms underlying masked and unmasked priming. Furthermore, it shed light on the mechanisms leading to the interindividual variation of priming effects as a function of the BDNF Val66Met polymorphism. General priming effects were mainly reflected by the drift rates $v$, supposedly representing semantic preactivation. Further priming processes could be mapped to the nondecision time $t 0$, and were especially pronounced in unmasked priming, suggesting additional conscious processes to be involved in the $t 0$ modulation, possibly semantic matching. Decreased decision thresholds $a$ in the nonrelated conditions may reflect a compensation for the lowered drift rates, in terms of a speed-accuracy trade-off to accomplish a comparable response time level within the whole experiment. The hypothesized superficial processing style of BDNF Val66Met Met+ carriers could be supported by the drift-diffusion analysis, following a similar speed-accuracy trade-off interpretation. We observed a lowered drift rate $v$ and a lowered decision threshold $a$ in the Met+-group. Although Met+ carriers showed decreased drift rates, they could achieve faster RTs by paying less attention to accuracy (decreased decision thresholds). Despite these differences in processing styles, semantic preactivation by a related prime, indexed by the semantic relatedness effect on drift rate $v$, was comparable in both genotype groups. Hence, this core mechanism of semantic priming does not seem to be altered in Met+ carriers. Overall, the present study adds further insight to the scarce literature considering the influence of semantic priming on drift-diffusion model parameters. Most importantly, we demonstrated that differences in cognitive tasks between genetic groups can be elucidated in more detail using drift-diffusion modeling.

\section{ACKNOWLEDGEMENTS}

This research was supported by the Deutsche Forschungsgemeinschaft (DFG, Kl 804/8-1).

\section{REFERENCES}

Barde, Y. A., Edgar, D., \& Thoenen, H. (1982). Purification of a new neurotrophic factor from mammalian brain. The EMBO Journal, 1,

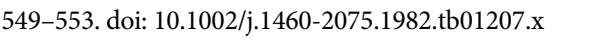

Beste, C., Schneider, D., Epplen, J. T., \& Arning, L. (2011). The functional BDNF Val66Met polymorphism affects functions of preattentive visual sensory memory processes. Neuropharmacology, 60, 467-471. doi: 10.1016/j.neuropharm.2010.10.028 السلسلس

Bodner, G. E., \& Masson, M. E. J. (2003). Beyond spreading activation: An influence of relatedness proportion on masked semantic priming. Psychonomic Bulletin and Review, 10, 645-652. doi: 10.3758/BF03196527 Wلس الس

Breitmeyer, B. (2007). Visual masking: Past accomplishments, present status, future developments. Advances in Cognitive Psychology, 3, 9-20 doi: 10.2478/v10053-008-0010-7 
Bueller, J. A., Aftab, M., Sen, S., Gomez-Hassan, D., Burmeister, M., \& Zubieta, J. K. (2006). BDNF Val66Met allele is associated with reduced hippocampal volume in healthy subjects. Biological Psychiatry, 9, 812-815. doi: 10.1016/j.biopsych.2005.09.022 الفالس

Carr, T. H., \& Dagenbach, D. (1990). Semantic priming and repetition priming from masked words: Evidence for a center-surround attentional mechanism in perceptual recognition. Journal of Experimental Psychology: Learning, Memory, and Cognition, 16, 341-350. doi: 10.1037/0278-7393.16.2.341 المالسلس

Collins, A. M., \& Loftus, E. F. (1975). A spreading-activation theory of semantic processing. Psychological Review, 82, 407-428. doi: 10.1037/0033-295X.82.6.407 سلس الس

Collins, A. M., \& Quillian, M. R. (1969). Retrieval time from semantic memory. Journal of Verbal Learning and Verbal Behavior, 8, 240-

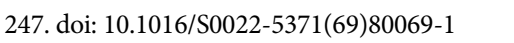

Cowles, M. K. (2013). Applied Bayesian statistics with R and OpenBUGS examples. Springer.

den Heyer, K., Briand, K., \& Smith, L. (1985). Automatic and strategic effects in semantic priming: An examination of Becker's verification model. Memory \& Cognition, 13, 228-232. doi: 10.3758/BF03197685 السلسلس

Durante, R., \& Hirshman, E. (1994). Retrospective priming and masked semantic priming: The interfering effects of prime activation. Journal of Memory and Language, 33, 112-127. doi: 10.1006/jmla.1994.1006 السلسلس Dutilh, G., Vandekerckhove, J., Forstmann, B. U., Keuleers, E., Brysbaert, M., \& Wagenmakers, E. J. (2012). Testing theories of post-error slowing. Attention, Perception, and Psychophysics, 74, 454-465. doi: 10.3758/s13414-011-0243-2 المالسلس

Egan, M. F., Kojima, M., Callicott, J. H., Goldberg, T. E., Kolachana, B. S., Bertolino, A., ... Weinberger, D. R. (2003). The BDNF Val66Met polymorphism affects activity-dependent secretion of BDNF and human memory and hippocampal function. Cell, 112, 257-269. doi: 10.1016/S0092-8674(03)00035-7 السلسلس

Fischler, I., \& Goodman, G. O. (1978). Latency of associative activation in memory. Journal of Experimental Psychology: Human Perception and Performance, 4, 455-470. doi: 10.1037/0096-1523.4.3.455 السلسلس

Gajewski, P. D., Hengstler, J. G., Golka, K., Falkenstein, M., \& Beste, C. (2011). The Met-allele of the BDNF Val66Met polymorphism enhances task switching in elderly. Neurobiology of Aging, 32, 2327. e7. doi: 10.1016/j.neurobiolaging.2011.06.010 الفيلسلس

Gajewski, P. D., Hengstler, J. G., Golka, K., Falkenstein, M., \& Beste, C. (2012). The Met-genotype of the BDNF Val66Met polymorphism is associated with reduced Stroop interference in elderly. Neuropsychologia,

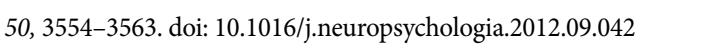

Gelman, A., \& Rubin, D. B. (1992). Inference from iterative simulation using multiple sequences. Statistical Science, 7, 457-472. doi: 10.1214/SS/1177011136 المسلس

Gomez, P., Perea, M., \& Ratcliff, R. (2013). A diffusion model account of masked versus unmasked priming: Are they qualitatively different? Journal of Experimental Psychology: Human Perception and Performance, 39, 1731-1740. doi: 10.1037/a0032333 السلسلسل

Green, D. G., \& Swets, J. A. (1966). Signal detection theory and psychophysics. Wiley.
Hall, D., Dhilla, A., Charalambous, A., Gogos, J. A., \& Karayiorgou, M. (2003). Sequence variants of the brain-derived neurotrophic factor (BDNF) gene are strongly associated with obsessive-compulsive disorder. American Journal of Human Genetics, 73, 370-376. doi: 10.1086/377003 السلسلس

Hänze, M., \& Hesse, F. W. (1993). Emotional influences on semantic priming. Cognition and Emotion, 7, 195-205. doi:

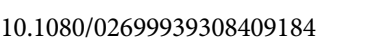

Harrisberger, F., Spalek, K., Smieskova, R., Schmidt, A., Coynel, D., Milnik, A. . . \& Borgwardt, S. (2014). The association of the BDNF Val66Met polymorphism and the hippocampal volumes in healthy humans: A joint meta-analysis of published and new data. Neuroscience and Biobehavioral Reviews, 42, 267-278. doi: 10.1016/j. neubiorev.2014.03.011 إلسلس

Heitz, R. P. (2014). The speed-accuracy tradeoff: History, physiology, methodology, and behavior. Frontiers in Neuroscience, 8, 150. doi: 10.3389/fnins.2014.00150 المسلس

Henson, R. N. A. (2003). Neuroimaging studies of priming. Progress in Neurobiology, 70, 53-81. doi: 10.1016/S0301-0082(03)00086-8 8 JASP Team. (2019). JASP. [Computer software]. السلسلس

Kambeitz, J. P., Bhattacharyya, S., Kambeitz-Ilankovic, L. M., Valli, I., Collier, D. A., \& McGuire, P. (2012). Effect of BDNF val66met polymorphism on declarative memory and its neural substrate: A metaanalysis. Neuroscience and Biobehavioral Reviews, 36, 2165-2177. doi: 10.1016/j.neubiorev.2012.07.002 السلسلس

Kerns, J. G., Cohen, J. D., MacDonald, A. W., Cho, R. Y., Stenger, V. A., \& Carter, C. S. (February, 2004). Anterior cingulate conflict monitoring and adjustments in control. Science, 303, 1023-1026. doi:

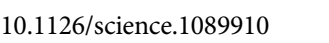

Kiefer, M. (2002). The N400 is modulated by unconsciously perceived masked words: further evidence for an automatic spreading activation account of N400 priming effects. Cognitive Brain Research, 13, 27-39. doi: 10.1016/S0926-6410(01)00085-4 المالسلسلئا

Kiefer, M., Ahlegian, M., \& Spitzer, M. (2005). Working memory capacity, indirect semantic priming, and Stroop interference: Pattern of interindividual prefrontal performance differences in healthy volunteers. Neuropsychology, 19, 332-344. doi: 10.1037/0894-4105.19.3.332 الملكلسلسا Kiefer, M., \& Brendel, D. (2006). Attentional modulation of unconscious "automatic" processes: Evidence from event-related potentials in a masked priming paradigm. Journal of Cognitive Neuroscience, 18, 184-198. doi: 10.1162/jocn.2006.18.2.184 المالسلسلكا

Kiefer, M., Harpaintner, M., Rohr, M., \& Wentura, D. (2020). Assessing subjective prime awareness on a trial-by-trial basis interferes with masked semantic priming effects. Manuscipt submitted for publication.

Kiefer, M., Martens, U., Weisbrod, M., Hermle, L., \& Spitzer, M. (2009). Increased unconscious semantic activation in schizophrenia patients with formal thought disorder. Schizophrenia Research, 114, 79-83. doi: 10.1016/j.schres.2009.07.024 المالملسلسا

Kiefer, M., \& Spitzer, M. (2000). Time course of conscious and unconscious semantic brain activation. NeuroReport, 11, 2401-2407. doi: 10.1097/00001756-200008030-00013 布

Klauer, K. C., \& Musch, J. (2002). Goal-dependent and goal-independent effects of irrelevant evaluations. Personality and Social Psychology 
Bulletin, 28, 802-814. doi: 10.1177/0146167202289009

Klauer, K. C., Voss, A., Schmitz, F., \& Teige-Mocigemba, S. (2007). Process components of the Implicit Association Test: A diffusionmodel analysis. Journal of Personality and Social Psychology, 93, 353-368. doi: 10.1037/0022-3514.93.3.353 المالسلس

Kwapil, T. R., Hegley, D. C., Chapman, L. J., \& Chapman, J. P. (1990). Facilitation of word recognition by semantic priming in schizophrenia. Journal of Abnormal Psychology, 99, 215-221. doi: 10.1037/0021-843X.99.3.215 الس الس

Lakens, D. (2013). Calculating and reporting effect sizes to facilitate cumulative science: A practical primer for t-tests and ANOVAs. Frontiers in Psychology, 4, 863. doi: 10.3389/fpsyg.2013.00863 المالسلسل|

Laroche, S., Davis, S., \& Jay, T. M. (2000). Plasticity at hippocampal to prefrontal cortex synapses: Dual roles in working memory and consolidation. Hippocampus, 10, 438-446. doi:

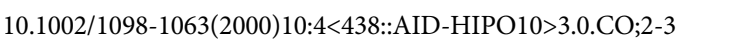

Lerche, V., Neubauer, A. B., \& Voss, A. (2018). Effects of implicit fear of failure on cognitive processing: A diffusion model analysis. Motivation and Emotion, 42, 386-402. doi: 10.1007/s11031-018-9691-5 المالسلسلس

Lerche, V., \& Voss, A. (2017). Retest reliability of the parameters of the Ratcliff diffusion model. Psychological Research, 81, 629-652. doi: 10.1007/s00426-016-0770-5 الفالسلس الس

Lu, B. (2003). BDNF and activity-dependent synaptic modulation. Learning and Memory, 10, 86-98. doi: 10.1101/lm.54603 المالسلس

Mandelman, S. D., \& Grigorenko, E. L. (2012). BDNF Val66Met and cognition: All, none, or some? A meta-analysis of the genetic association. Genes, Brain and Behavior, 11, 127-136. doi: 10.1111/j.1601183X.2011.00738.x السلسلس

Martinowich, K., Manji, H., \& Lu, B. (2007). New insights into BDNF function in depression and anxiety. Nature Neuroscience, 10, 10891093. doi: 10.1038/nn1971 |لس الس

Masson, M. E. J. (1995). A distributed memory model of semantic priming. Journal of Experimental Psychology: Learning, Memory, and Cognition, 21, 3-23. doi: 10.1037/0278-7393.21.1.3 المالسلسلس

McNamara, T. P. (1992). Theories of priming: I. Associative distance and lag. Journal of Experimental Psychology: Learning, Memory, and Cognition, 18, 1173-1190. doi: 10.1037/0278-7393.18.6.1173 السلسلس

Merikle, P. M., Joordens, S., \& Stolz, J. A. (1995). Measuring the relative magnitude of unconscious influences. Consciousness and Cognition, 4, 422-439. doi: 10.1006/ccog.1995.1049 المالسلسلة

Meyer, D. E., \& Schvaneveldt, R. W. (1971). Facilitation in recognizing pairs of words: Evidence of a dependence between retrieval operations. Journal of Experimental Psychology, 90, 227-234. doi: 10.1037/h0031564 السلسلكالس

Montag, C., Basten, U., Stelzel, C., Fiebach, C. J., \& Reuter, M. (2010). The BDNF Val66Met polymorphism and anxiety: Support for animal knock-in studies from a genetic association study in humans. Psychiatry Research, 179, 86-90. doi: 10.1016/J.

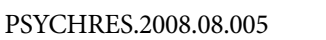

Montag, C., Ebstein, R., Jawinski, P., \& Markett, S. (2020). Molecular genetics in psychology and personality neuroscience: On candidate genes, genome wide scans, and new research strategies. Neuroscience \& Biobehavioral Reviews, 118, 163-174. doi: 10.1016/j. neubiorev.2020.06.020
Montag, C., Felten, A., Markett, S., Fischer, L., Winkel, K., Cooper, A., \& Reuter, M. (2014). The role of the BDNF Val66Met polymorphism in individual differences in long-term memory capacity. Journal of Molecular Neuroscience, 54, 796-802. doi: 10.1007/s12031-014-0417-1 السالسلس الس

Montag, C., \& Reuter, M. (2014). Disentangling the molecular genetic basis of personality: From monoamines to neuropeptides. Neuroscience \& Biobehavioral Reviews, 43, 228-239. doi: 10.1016/J. NEUBIOREV.2014.04.006 الس الس الس

Montag, C., Schoene-Bake, J.-C., Faber, J., Reuter, M., \& Weber, B. (2010). Genetic variation on the BDNF gene is not associated with differences in white matter tracts in healthy humans measured by tract-based spatial statistics. Genes, Brain and Behavior, 9, 886-891. doi: 10.1111/j.1601-183X.2010.00626.x السلسلس

Montag, C., Weber, B., Fliessbach, K., Elger, C., \& Reuter, M. (2009). The BDNF Val66Met polymorphism impacts parahippocampal and amygdala volume in healthy humans: incremental support for a genetic risk factor for depression. Psychological Medicine, 39, 1831-1839. doi: 10.1017/S0033291709005509 الس السلسل

Moritz, S., Andresen, B., Domin, F., Martin, T., Probsthein, E., Kretschmer, G., Krausz, M., . . \& \& Spitzer, M. (1999). Increased automatic spreading activation in healthy subjects with elevated scores in a scale assessing schizophrenic language disturbances. Psychological Medicine, 29, 161-170. doi: 10.1017/S0033291798007831 السلسلس

Moritz, S., Woodward, T. S., Küppers, D., Lausen, A., \& Schickel, M. (2003). Increased automatic spreading of activation in thoughtdisordered schizophrenic patients. Schizophrenia Research, 59, 181-186. doi: 10.1016/S0920-9964(01)00337-1 المالسلس

Neely, J. H. (1991). Semantic priming effects in visual word recognition: A selective review of current findings and theories. In D. Besner, \& G. W. Humphreys (Eds.), Basic processes in reading: Visual word recognition (pp. 264-337). Lawrence Erlbaum Associated.

Neely, J. H. (1976). Semantic priming and retrieval from lexical memory: Evidence for facilitatory and inhibitory processes. Memory \& Cognition, 4, 648-654. doi: 10.3758/BF03213230 الملسلس

Neely, J. H. (1977). Semantic priming and retrieval from lexical memory: Roles of inhibitionless spreading activation and limitedcapacity attention. Journal of Experimental Psychology: General, 106, 226-254. doi: 10.1037/0096-3445.106.3.226 المسلسلس

Neely, J. H., Keefe, D. E., \& Ross, K. L. (1989). Semantic Priming in the Lexical Decision Task: Roles of prospective prime-generated expectancies and retrospective semantic matching. Journal of Experimental Psychology: Learning, Memory, and Cognition, 15, 1003-1019. doi: 10.1037/0278-7393.15.6.1003 الس السلس

Norman, D. A., \& Shallice, T. (1986). Attention to action. In R. J. Davidson, G. E. Schwartz, \& D. Shapiro (Eds.), Consciousness and self-regulation (pp. 1-18). Springer.

Oldfield, R. C. (1971). The assessment and analysis of handedness: the Edinburgh inventory. Neuropsychologia, 9, 97-113.

Pezawas, L., Verchinski, B. A., Mattay, V. S., Callicott, J. H., Kolachana, B. S., Straub, R. E., .. . \& Weinberger, D. R. (2004). The brain-derived neurotrophic factor val66met polymorphism and variation in human cortical morphology. Journal of Neuroscience, 24, 10099-10102. 


\section{doi: 10.1523/JNEUROSCI.2680-04.2004 |ل|}

Plaut, D. C. (1995). Semantic and associative priming in a distributed attractor network. In J. D. Moore \& J. . Lehman (Eds.), Proceedings of the 17th Annual Conference of the Cognitive Science Society (pp. 37-43). Cognitive Science Society.

Posner, M. I., \& Snyder, C. R. (1975). Attention and cognitive control. In R. Solso (Ed.), Information processing and cognition: The Loyola symposium (pp. 55-85). Lawrence Erlbaum Associated.

Ratcliff, R. (1978). A theory of memory retrieval. Psychological Review. 85, 59-108. doi: 10.1037/0033-295X.85.2.59 المالسلسلة

Ratcliff, R., \& McKoon, G. (1988). A retrieval theory of priming in memory. Psychological Review, 95, 385-408. doi: 10.1037/0033-295X.95.3.385 المالسلس

Ratcliff, R., \& McKoon, G. (2008). The diffusion decision model: Theory and data for two-choice decision tasks. Neural Computation, 20, 873-922. doi: 10.1162/neco.2008.12-06-420 السلسلسل

Ratcliff, R., Spieler, D., \& Mckoon, G. (2000). Explicitly modeling the effects of aging on response time. Psychonomic Bulletin and Review,

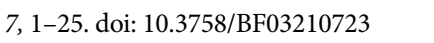

Ratcliff, R., Thapar, A., \& McKoon, G. (2006). Aging and individual differences in rapid two-choice decisions. Psychonomic Bulletin and Review, 13, 626-635. doi: 10.3758/BF03193973 سلس

Reuter, M., Montag, C., Peters, K., Kocher, A., \& Kiefer, M. (2009). The modulatory influence of the functional COMT Val158Met polymorphism on lexical decisions and semantic priming. Frontiers in Human Neuroscience, 3, 20. doi: 10.3389/neuro.09.020.2009 السلسلس

Rouder, J. N., Morey, R. D., Speckman, P. L., \& Province, J. M. (2012). Default Bayes factors for ANOVA designs. Journal of Mathematical Psychology, 56, 356-374. doi: 10.1016/j.jmp.2012.08.001 المبلسلس

Sanwald, S., Montag, C., \& Kiefer, M. (2020). Depressive emotionality moderates the influence of the BDNF Val66Met polymorphism on executive functions and on unconscious semantic priming. Journal of Molecular Neuroscience, 70, 699-712. doi: 10.1007/s12031-020-01479-x المالسلسليكا

Schmiedek, F., Oberauer, K., Wilhelm, O., Süß, H. M., \& Wittmann, W. W. (2007). Individual differences in components of reaction time distributions and their relations to working memory and intelligence. Journal of Experimental Psychology: General, 136, 414-429. doi: 10.1037/0096-3445.136.3.414 سلس

Schmitz, F., \& Voss, A. (2012). Decomposing task-switching costs with the diffusion model. Journal of Experimental Psychology: Human Perception and Performance, 38, 222-250. doi: 10.1037/a0026003 المالسلس

Seidenberg, M. S., Waters, G. S., Sanders, M., \& Langer, P. (1984). Pre- and postlexical loci of contextual effects on word recognition.

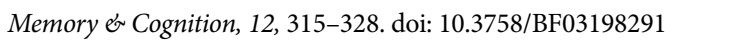

Shimizu, E., Hashimoto, K., \& Iyo, M. (2004). Ethnic difference of the BDNF 196G/A (val66met) polymorphism frequencies: The possibility to explain ethnic mental traits. American Journal of Medical Genetics, 126B, 122-123. doi: 10.1002/ajmg.b.20118 السلسلسلاس

Spiegelhalter, D. J., Best, N. G., Carlin, B. P., \& Van Der Linde, A. (2002). Bayesian measures of model complexity and fit. Journal of the Royal Statistical Society. Series B: Statistical Methodology, 64, 583-639. doi: 10.1111/1467-9868.00353 سلس

Spitzer, M., Braun, U., Hermle, L., \& Maier, S. (1993). Associative se- mantic network dysfunction in thought-disordered schizophrenic patients: Direct evidence from indirect semantic priming. Biological Psychiatry, 34, 864-877. doi: 10.1016/0006-3223(93)90054-H المالسليلكا

Ulrich, M., Adams, S. C., \& Kiefer, M. (2014). Flexible establishment of functional brain networks supports attentional modulation of unconscious cognition. Human Brain Mapping, 35, 5500-5516. doi:

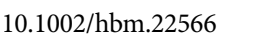

Ulrich, M., Hoenig, K., Grön, G., \& Kiefer, M. (2013). Brain activation during masked and unmasked semantic priming: Commonalities and differences. Journal of Cognitive Neuroscience, 25, 2216-2229.

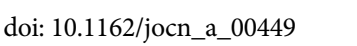

Voss, A., Nagler, M., \& Lerche, V. (2013). Diffusion models in experimental psychology: A practical introduction. Experimental

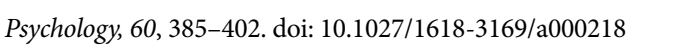

Voss, A., Rothermund, K., Gast, A., \& Wentura, D. (2013). Cognitive processes in associative and categorical priming: A diffusion model analysis. Journal of Experimental Psychology: General, 142, 536-559. doi: 10.1037/a0029459 1لس

Voss, A., Rothermund, K., \& Voss, J. (2004). Interpreting the parameters of the diffusion model: An empirical validation. Memory \& Cognition, 32, 1206-1220. doi: 10.3758/BF03196893 الملسلسلس

Wagenmakers, E. J., Love, J., Marsman, M., Jamil, T., Ly, A., Verhagen, J., ... Morey, R. D. (2018). Bayesian inference for psychology. Part II: Example applications with JASP. Psychonomic Bulletin and Review, 25, 58-76. doi: 10.3758/s13423-017-1323-7 المالسلسلة

Wagner, A. D., Paré-Blagoev, E. J., Clark, J., \& Poldrack, R. A. (2001). Recovering meaning: Left prefrontal cortex guides controlled semantic retrieval. Neuron, 32, 329-338. doi: 10.1016/S08966273(01)00359-2 布

Wang, Q., Liu, J., Guo, Y., Dong, G., Zou, W., \& Chen, Z. (2019). Association between BDNF G196A (Val66Met) polymorphism and cognitive impairment in patients with Parkinson's disease: A metaanalysis. Brazilian Journal of Medical and Biological Research, 52. doi: 10.1590/1414-431x20198443 السلس

Wentura, D. (2000). Dissociative affective and associative priming effects in the lexical decision task: Yes versus no responses to word targets reveal evaluative judgment tendencies. Journal of Experimental Psychology: Learning Memory \& Cognition, 26, 456-469. doi: 10.1037/0278-7393.26.2.456 الس

Wentura, D., Rohr, M., \& Kiefer, M. (2020). Does affective processing require awareness? On the use of the perceptual awareness scale in response priming research. Manuscipt submitted for publication.

Wiecki, T. V., Sofer, I., \& Frank, M. J. (2013). HDDM: Hierarchical bayesian estimation of the drift-diffusion model in Python. Frontiers in Neuroinformatics, 7, 14. doi: 10.3389/fninf.2013.00014 الس الس

Yin, Y., Su, X., Pan, L., \& Li, C. (2019). BDNF Val66Met polymorphism and cognitive impairment in Parkinson's disease-a meta-analysis. Neurological Sciences, 40, 1901-1907. doi: 10.1007/s10072-019-03907-2 Шالس

RECEIVED 10.09.2020 | ACCEPTED 15.01.2021 


\section{SUPPLEMENTARY MATERIAL}

\section{A: Evaluation of Drift-Diffusion Model Convergence}

The HDDM toolbox (Wiecki et al., 2013) provides several methods to assess model convergence. Assessing Markov chain Monte Carlo (MCMC) chain convergence is crucial to "ensure that we are sampling from the actual posterior distribution"1. For this study, model convergence was inspected by: (a) evaluating the Monte Carlo (MC) error statistic, (b) visually inspecting the MCMC chains, and (c) calculating the GelmanRubin statistic. Model convergence was assessed separately for the driftdiffusion models of the masked and unmasked priming paradigm.

\section{EVALUATION OF THE MONTE CARLO ERROR STATISTIC}

For every estimated parameter, a Monte Carlo (MC) error statistic is provided. The MC error indicates the amount of uncertainty of the sampling process (Cowles, 2013). Cowles (2013) suggests the criterion of an $\mathrm{MC}$ error not larger than $5 \%$ of the posterior $S D, \mathrm{MC}$ error $\leq 0.05 \times$ posterior SD. For the masked priming paradigm, every MC error statistic fulfilled this criterion. Considering the unmasked priming paradigm, 99.7\% of the MC error statistics fulfilled this criterion.

\section{VISUAL INSPECTION OF THE CHAINS}

The HDDM provides for every estimated (group) parameter the posterior trace, the autocorrelation, and the posterior histogram (for details see Wiecki et al., 2013). Appropriate convergence should be characterized by the absence of drifts or large jumps in the trace, an early drop of the autocorrelation to about zero and the histogram should look "smooth" (like a normal distribution for group mean parameters and a gamma distribution for group variability parameters, Wiecki et al., 2013). To provide an overview of the traces, autocorrelations and posterior histograms, the (subjectively evaluated) best and worst converged examples are presented. The best example (see Figure A1) shows a smooth trace, no autocorrelation, and a normal-distributed histogram. In contrast, the worst example (see Figure A2) shows some jumps in the trace (but they are considerably small) and a relatively late drop of the autocorrelation to near zero. Nevertheless, the histogram appears appropriate. To summarize, even the worst example seems to have converged ${ }^{2}$, even if the plots look clearly less unproblematic than the best example.

\section{GELMAN-RUBIN STATISTIC}

The Gelman-Rubin statistic compares the within-chain with the between-chain variance for different iterations of the same model (Gelman \& Rubin, 1992). Therefore, values close to 1 indicate that between-chain and within-chain variance are (nearly) equal, that is, the sampling process did not differ between iterations. This indicates that the MCMC chains have converged. Considering the Gelman-Rubin statistic, Wiecki et al. (2013) suggest a deviation of less than 0.02 from 1, that is, $1.02>$ Gelman-Rubin statistic $>0.98$, as indicator for appropriate convergence. Considering the masked as well as the unmasked priming paradigm, every Gelman-Rubin statistic fulfilled this criterion. A summary of the Gelman-Rubin statistics is provided in Table A1.

\section{SUMMARY}

Summarizing the three different evaluation methods of model convergence, model convergence can be evaluated as satisfying. None of the different methods indicated crucial convergence problems. The visual inspection indicated differences in the quality of model convergence. Nevertheless, all model seemed to have converged as indicated by the MC error and Gelman-Rubin statistics.

\section{B: Comparison of the Posterior Distributions of Drift-Diffusion Model Parameters}

We compared the posterior distributions of the drift-diffusion model group parameters to assess which experimental conditions differed. In the Bayesian approach used, there is no need to calculate additional significance tests to compare conditions, as the whole distribution of a parameter estimation (posterior distribution) is available for use. Therefore, different conditions can be directly tested against each other using their posterior distributions (Wiecki et al., 2013).

We performed posterior comparisons for all conditions ${ }^{3}$ of the driftdiffusion models of the masked and unmasked priming paradigm. Those comparisons and respective probabilities (p[parameter $A>$ parameter $B$ ] or [parameter $A<$ parameter $B]$ ) are presented in the following sections. The following abbreviations are used:

-cond_A / cond_B: The conditions which are tested against each other. The first letter indicates the drift-diffusion model parameter. $\mathrm{v}=$ drift rate $\mathrm{v}, \mathrm{a}=$ decision threshold $\mathrm{a}, \mathrm{t}=$ nondecision time $\mathrm{t} 0$. The condition is specified in parentheses. $\mathrm{A}$ = related condition (associated), $\mathrm{I}$ = indirectly related condition, $\mathrm{N}=$ nonrelated condition. met $=$ Met + genotype group, $\mathrm{val}=\mathrm{Val} / \mathrm{Val}$ genotype group .

-m_A / m_B: Mean of the respective parameter in Condition A and Condition B.

-sd_A / sd_B: SD of the respective parameter in Condition A and Condition B.

$-\mathrm{p}(\mathrm{A}<\mathrm{B}) / \mathrm{p}(\mathrm{A}>\mathrm{B})$ : Posterior probability of the parameter in Condition A being greater / smaller than the parameter in Condition $\mathrm{B}$. As $p(A<B)=1-p(A>B)$ (and vice versa), only one of both probabilities is given.

The data is presented in Tables A2-A3.

\section{REFERENCES}

Cowles, M. K. (2013). Applied Bayesian Statistics With R and OpenBUGS Examples. In Springer Texts in Statistics,.

Gelman, A., \& Rubin, D. B. (1992). Inference from Iterative Simulation Using Multiple Sequences. Statistical Science, 7(4), 457-472. https:// doi.org/10.1214/SS/1177011136

Wiecki, T. V., Sofer, I., \& Frank, M. J. (2013). HDDM: Hierarchical bayesian estimation of the drift-diffusion model in Python. Frontiers in Neuroinformatics. https://doi.org/10.3389/fninf.2013.00014 

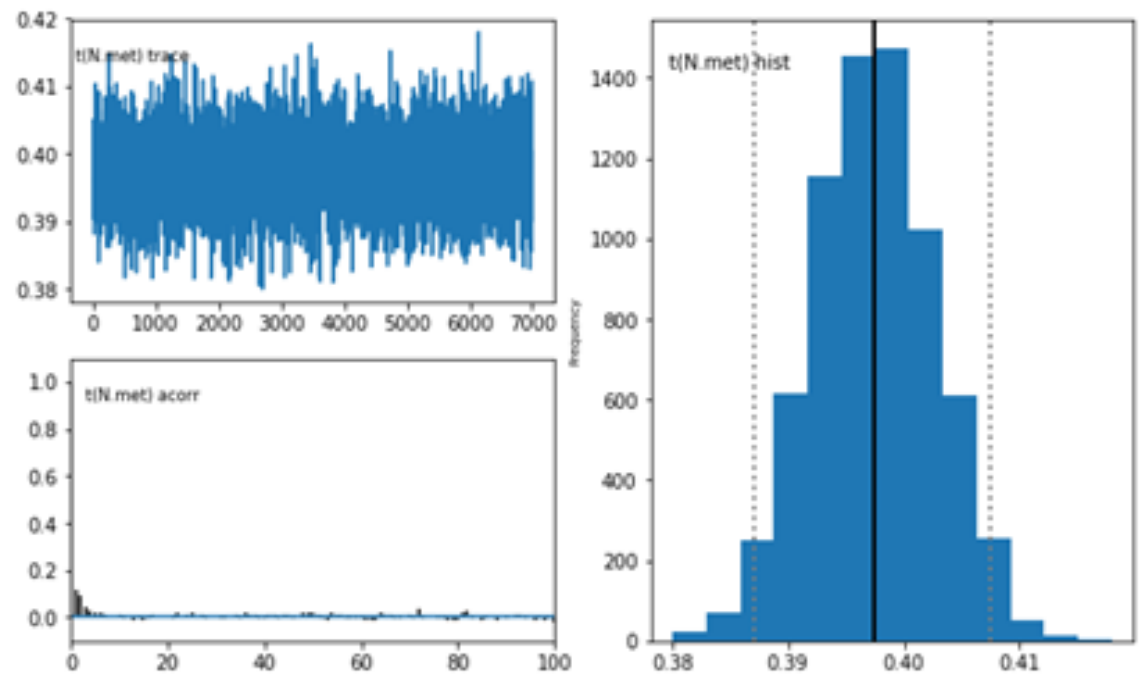

FIGURE A1.

Visual indicators of model convergence for the best converged example. The parameter shown is to for the condition non-related prime; Met+ group in the masked priming paradigm.
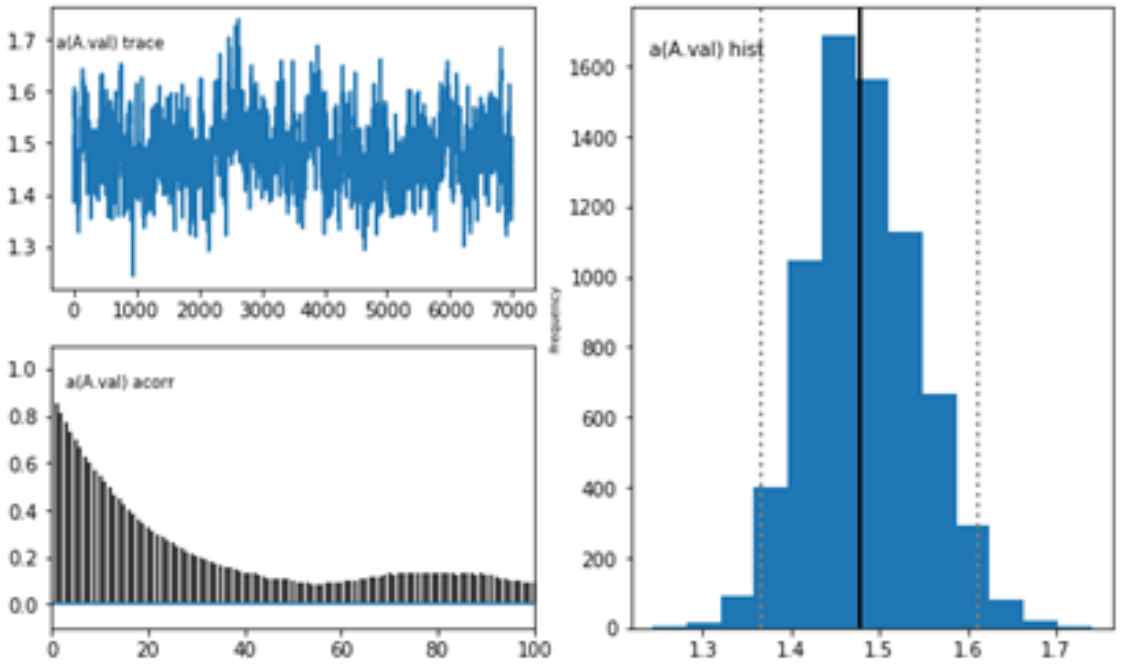

FIGURE A2.

Visual indicators of model convergence for the worst converged example. The parameter shown is a for the condition related prime; $V$ al $N$ al group in the unmasked priming paradigm.

\footnotetext{
TABLE A1.

Summary of the Gelman-Rubin Statistics of the Masked and Unmasked Priming Paradigm

\begin{tabular}{ccccc}
\hline Paradigm & $\mathrm{M}$ & $\mathrm{SD}$ & Min. & Max. \\
\hline masked & 1.00072 & 0.0006 & 1.0001 & 1.0022 \\
unmasked & 1.00300 & 0.0035 & 0.9999 & 1.0116
\end{tabular}
}




\section{TABLE A2. \\ Masked priming paradigm}

\begin{tabular}{|c|c|c|c|c|c|c|c|}
\hline \multicolumn{8}{|c|}{ Parameter $v$} \\
\hline cond_A & cond_B & m_A & m_B & sd_A & sd_B & $\mathrm{p}(\mathrm{A}<\mathrm{B})$ & $\mathrm{p}(\mathrm{A}>\mathrm{B})$ \\
\hline $\mathrm{v}$ (A.met) & $\mathrm{v}($ A.val $)$ & 3.7941 & 4.0021 & 0.107 & 0.086 & 0.9360 & - \\
\hline $\mathrm{v}$ (A.met) & $\mathrm{v}$ (N.met) & 3.7941 & 3.1930 & 0.107 & 0.100 & - & 1.0000 \\
\hline $\mathrm{v}$ (A.met) & v(N.val) & 3.7941 & 3.4429 & 0.107 & 0.079 & - & 0.9969 \\
\hline $\mathrm{v}($ A.val $)$ & $\mathrm{v}$ (N.met) & 4.0021 & 3.1930 & 0.086 & 0.100 & - & 1.0000 \\
\hline $\mathrm{v}($ A.val $)$ & $\mathrm{v}(\mathrm{N} . v a l)$ & 4.0021 & 3.4429 & 0.086 & 0.079 & - & 1.0000 \\
\hline $\mathrm{v}$ (N.met) & $\mathrm{v}(\mathrm{N} . v a l)$ & 3.1930 & 3.4429 & 0.100 & 0.079 & 0.9794 & - \\
\hline \multicolumn{8}{|c|}{ Parameter $a$} \\
\hline cond_A & cond_B & m_A & m_B & sd_A & sd_B & $\mathrm{p}(\mathrm{A}<\mathrm{B})$ & $\mathrm{p}(\mathrm{A}>\mathrm{B})$ \\
\hline a(A.met) & a(A.val) & 1.3700 & 1.4465 & 0.046 & 0.039 & 0.9026 & - \\
\hline a(A.met) & $\mathrm{a}(\mathrm{N} . \mathrm{met})$ & 1.3700 & 1.2345 & 0.046 & 0.039 & - & 0.9904 \\
\hline a(A.met) & $\mathrm{a}(\mathrm{N} . v a l)$ & 1.3700 & 1.3957 & 0.046 & 0.035 & 0.6850 & - \\
\hline a(A.val) & $\mathrm{a}($ N.met $)$ & 1.4465 & 1.2345 & 0.039 & 0.039 & - & 1.0000 \\
\hline a(A.val) & $\mathrm{a}(\mathrm{N} . v a l)$ & 1.4465 & 1.3957 & 0.039 & 0.035 & - & 0.8486 \\
\hline a(N.met) & a(N.val) & 1.2345 & 1.3957 & 0.039 & 0.035 & 0.9994 & - \\
\hline \multicolumn{8}{|c|}{ Parameter $t 0$} \\
\hline cond_A & cond_B & m_A & m_B & sd_A & sd_B & $\mathrm{p}(\mathrm{A}<\mathrm{B})$ & $\mathrm{p}(\mathrm{A}>\mathrm{B})$ \\
\hline $\mathrm{t}($ A.met $)$ & $\mathrm{t}$ (A.val) & 0.3850 & 0.3892 & 0.005 & 0.004 & 0.7304 & - \\
\hline$t$ (A.met) & $\mathrm{t}$ (N.met) & 0.3850 & 0.3974 & 0.005 & 0.005 & 0.9493 & - \\
\hline $\mathrm{t}$ (A.met) & $\mathrm{t}(\mathrm{N} . v a l)$ & 0.3850 & 0.3938 & 0.005 & 0.004 & 0.9017 & - \\
\hline $\mathrm{t}$ (A.val) & $\mathrm{t}(\mathrm{N} \cdot \mathrm{met})$ & 0.3892 & 0.3974 & 0.004 & 0.005 & 0.8900 & - \\
\hline $\mathrm{t}$ (A.val) & $\mathrm{t}(\mathrm{N}$.val $)$ & 0.3892 & 0.3938 & 0.004 & 0.004 & 0.7833 & - \\
\hline $\mathrm{t}($ N.met $)$ & $\mathrm{t}$ (N.val) & 0.3974 & 0.3938 & 0.005 & 0.004 & - & 0.7084 \\
\hline
\end{tabular}

TABLE A3.

Unmasked priming paradigm

\begin{tabular}{|c|c|c|c|c|c|c|c|}
\hline \multicolumn{8}{|c|}{ Parameter $v$} \\
\hline cond_A & cond_B & m_A & m_B & sd_A & sd_B & $\mathrm{p}(\mathrm{A}<\mathrm{B})$ & $\mathrm{p}(\mathrm{A}>\mathrm{B})$ \\
\hline $\mathrm{v}$ (A.met) & $\mathrm{v}$ (A.val) & 4.4170 & 4.4261 & 0.173 & 0.137 & 0.5153 & - \\
\hline $\mathrm{v}$ (A.met) & $\mathrm{v}$ (I.met) & 4.4170 & 3.5195 & 0.173 & 0.144 & - & 1.0000 \\
\hline $\mathrm{v}$ (A.met) & $\mathrm{v}(\mathrm{I} . v a l)$ & 4.4170 & 3.8078 & 0.173 & 0.116 & - & 0.9994 \\
\hline $\mathrm{v}$ (A.met) & $\mathrm{v}$ (N.met) & 4.4170 & 2.9452 & 0.173 & 0.132 & - & 1.0000 \\
\hline $\mathrm{v}$ (A.met) & $\mathrm{v}(\mathrm{N} . \mathrm{val})$ & 4.4170 & 3.1303 & 0.173 & 0.107 & - & 1.0000 \\
\hline $\mathrm{v}($ A.val $)$ & v(I.met) & 4.4261 & 3.5195 & 0.137 & 0.144 & - & 1.0000 \\
\hline $\mathrm{v}$ (A.val) & $\mathrm{v}(\mathrm{I} . \mathrm{val})$ & 4.4261 & 3.8078 & 0.137 & 0.116 & - & 0.9994 \\
\hline $\mathrm{v}($ A.val) & $\mathrm{v}$ (N.met) & 4.4261 & 2.9452 & 0.137 & 0.132 & - & 1.0000 \\
\hline $\mathrm{v}($ A.val $)$ & $\mathrm{v}(\mathrm{N} . v a l)$ & 4.4261 & 3.1303 & 0.137 & 0.107 & - & 1.0000 \\
\hline $\mathrm{v}$ (I.met) & $\mathrm{v}(\mathrm{I} . v a l)$ & 3.5195 & 3.8078 & 0.144 & 0.116 & 0.9440 & - \\
\hline $\mathrm{v}$ (I.met) & $\mathrm{v}$ (N.met) & 3.5195 & 2.9452 & 0.144 & 0.132 & - & 0.9987 \\
\hline v(I.met) & $\mathrm{v}(\mathrm{N} . \mathrm{val})$ & 3.5195 & 3.1303 & 0.144 & 0.107 & - & 0.9856 \\
\hline $\mathrm{v}(\mathrm{I} . \mathrm{val})$ & $\mathrm{v}$ (N.met) & 3.8078 & 2.9452 & 0.116 & 0.132 & - & 1.0000 \\
\hline $\mathrm{v}$ (I.val) & $\mathrm{v}(\mathrm{N} . v a l)$ & 3.8078 & 3.1303 & 0.116 & 0.107 & - & 1.0000 \\
\hline $\mathrm{v}$ (N.met) & $\mathrm{v}(\mathrm{N} . v a l)$ & 2.9452 & 3.1303 & 0.132 & 0.107 & 0.8720 & - \\
\hline \multicolumn{8}{|c|}{ Parameter $a$} \\
\hline cond_A & cond_B & m_A & m_B & sd_A & sd_B & $\mathrm{p}(\mathrm{A}<\mathrm{B})$ & $\mathrm{p}(\mathrm{A}>\mathrm{B})$ \\
\hline a(A.met) & a(A.val) & 1.4827 & 1.4826 & 0.075 & 0.063 & - & 0.5149 \\
\hline a(A.met) & $\mathrm{a}$ (I.met) & 1.4827 & 1.3012 & 0.075 & 0.054 & - & 0.9779 \\
\hline a(A.met) & $\mathrm{a}(\mathrm{I} . v a l)$ & 1.4827 & 1.4408 & 0.075 & 0.051 & - & 0.6797 \\
\hline a(A.met) & $\mathrm{a}(\mathrm{N} . \mathrm{met})$ & 1.4827 & 1.1447 & 0.075 & 0.044 & - & 1.0000 \\
\hline a(A.met) & $\mathrm{a}(\mathrm{N} . v a l)$ & 1.4827 & 1.2420 & 0.075 & 0.038 & - & 0.9991 \\
\hline a(A.val) & a(I.met) & 1.4826 & 1.3012 & 0.063 & 0.054 & - & 0.9916 \\
\hline a(A.val) & a(I.val) & 1.4826 & 1.4408 & 0.063 & 0.051 & - & 0.7010 \\
\hline a(A.val) & $\mathrm{a}(\mathrm{N} . \mathrm{met})$ & 1.4826 & 1.1447 & 0.063 & 0.044 & - & 1.0000 \\
\hline $\mathrm{a}($ A.val $)$ & $\mathrm{a}(\mathrm{N} . v a l)$ & 1.4826 & 1.2420 & 0.063 & 0.038 & - & 0.9994 \\
\hline $\mathrm{a}$ (I.met) & $\mathrm{a}(\mathrm{I} . v a l)$ & 1.3012 & 1.4408 & 0.054 & 0.051 & 0.9744 & - \\
\hline a(I.met) & $\mathrm{a}(\mathrm{N} . \mathrm{met})$ & 1.3012 & 1.1447 & 0.054 & 0.044 & - & 0.9916 \\
\hline a(I.met) & $\mathrm{a}(\mathrm{N} . v a l)$ & 1.3012 & 1.2420 & 0.054 & 0.038 & - & 0.8237 \\
\hline $\mathrm{a}$ (I.val) & $\mathrm{a}(\mathrm{N} . \mathrm{met})$ & 1.4408 & 1.1447 & 0.051 & 0.044 & - & 1.0000 \\
\hline a(I.val) & $\mathrm{a}(\mathrm{N} . v a l)$ & 1.4408 & 1.2420 & 0.051 & 0.038 & - & 0.9996 \\
\hline $\mathrm{a}($ N.met) & $\mathrm{a}($ N.val) & 1.1447 & 1.2420 & 0.044 & 0.038 & 0.9611 & - \\
\hline \multicolumn{8}{|c|}{ Parameter $t 0$} \\
\hline cond_A & cond_B & m_A & m_B & sd_A & sd_B & $\mathrm{p}(\mathrm{A}<\mathrm{B})$ & $\mathrm{p}(\mathrm{A}>\mathrm{B})$ \\
\hline$t$ (A.met) & $\mathrm{t}($ A.val $)$ & 0.3617 & 0.3692 & 0.008 & 0.006 & 0.7841 & - \\
\hline $\mathrm{t}$ (A.met) & $\mathrm{t}$ (I.met) & 0.3617 & 0.3816 & 0.008 & 0.007 & 0.9731 & - \\
\hline$t$ (A.met) & $\mathrm{t}(\mathrm{I} . \mathrm{val})$ & 0.3617 & 0.3860 & 0.008 & 0.006 & 0.9947 & - \\
\hline$t$ (A.met) & $\mathrm{t}$ (N.met) & 0.3617 & 0.3999 & 0.008 & 0.007 & 1.0000 & - \\
\hline$t$ (A.met) & $\mathrm{t}(\mathrm{N} . v a l)$ & 0.3617 & 0.3998 & 0.008 & 0.005 & 1.0000 & - \\
\hline $\mathrm{t}($ A.val $)$ & $\mathrm{t}$ (I.met) & 0.3692 & 0.3816 & 0.006 & 0.007 & 0.9153 & - \\
\hline $\mathrm{t}($ A.val $)$ & $\mathrm{t}(\mathrm{I} . \mathrm{val})$ & 0.3692 & 0.3860 & 0.006 & 0.006 & 0.9789 & - \\
\hline $\mathrm{t}$ (A.val) & $\mathrm{t}(\mathrm{N} . \mathrm{met})$ & 0.3692 & 0.3999 & 0.006 & 0.007 & 0.9997 & - \\
\hline $\mathrm{t}$ (A.val) & $\mathrm{t}(\mathrm{N} . v a l)$ & 0.3692 & 0.3998 & 0.006 & 0.005 & 0.9999 & - \\
\hline$t($ I.met $)$ & $\mathrm{t}(\mathrm{I} . \mathrm{val})$ & 0.3816 & 0.3860 & 0.007 & 0.006 & 0.6911 & - \\
\hline$t($ I.met $)$ & $\mathrm{t}(\mathrm{N} \cdot \mathrm{met})$ & 0.3816 & 0.3999 & 0.007 & 0.007 & 0.9711 & - \\
\hline $\mathrm{t}($ I.met $)$ & $\mathrm{t}(\mathrm{N} . v a l)$ & 0.3816 & 0.3998 & 0.007 & 0.005 & 0.9809 & - \\
\hline $\mathrm{t}(\mathrm{I} . \mathrm{val})$ & $\mathrm{t}$ (N.met) & 0.3860 & 0.3999 & 0.006 & 0.007 & 0.9387 & - \\
\hline $\mathrm{t}(\mathrm{I} . v a l)$ & $\mathrm{t}($ N.val) & 0.3860 & 0.3998 & 0.006 & 0.005 & 0.9603 & - \\
\hline $\mathrm{t}(\mathrm{N} . \mathrm{met})$ & $\mathrm{t}($ N.val) & 0.3999 & 0.3998 & 0.007 & 0.005 & - & 0.5043 \\
\hline
\end{tabular}

\section{FOOTNOTES}

${ }^{1}$ HDDM-Wiki: http://ski.clps.brown.edu/hddm_docs/howto.html\#assess-model-convergence

${ }^{2}$ For an example of a non converged chain, see: http://ski.clps.brown.edu/hddm_docs/howto.html\#assess-model-convergence

${ }^{3}$ The parameters provided here are the group nodes, that is, the estimated mean parameters for that condition. Note that these are not the same parameters as the average of the subject parameters used for the Bayesian analyses of variance (ANOVAs) analysis in the paper. 